\title{
Hydrogel Films on Optical Fiber Core: Properties, Challenges, and Prospects for Future Applications
}

Sergey V. Kazakov

Additional information is available at the end of the chapter

http://dx.doi.org/10.5772/48371

\section{Introduction}

\subsection{Properties of hydrogels}

Gels are most generally understood to be 3D-polymeric networks with cross-linked long chain molecules, which absorb a large amount of solvent to cause macroscopic changes in dimensions. Polymer network immersed in an aqueous medium is referred to as a hydrogel. The three-dimensional network of a hydrogel can be stabilized as a gigantic single molecule by chemical (covalent bonds) or/and physical (ionic bonds, entanglements, crystallites, charge complexes, hydrogen bonding, van der Waals or hydrophobic interactions) crosslinks. A hydrogel is also considered as an open container with semipermeable boundaries, across which water and solute molecules can move whereas charged (ionizable) groups fixed on the network chains cannot move (Figure 1).

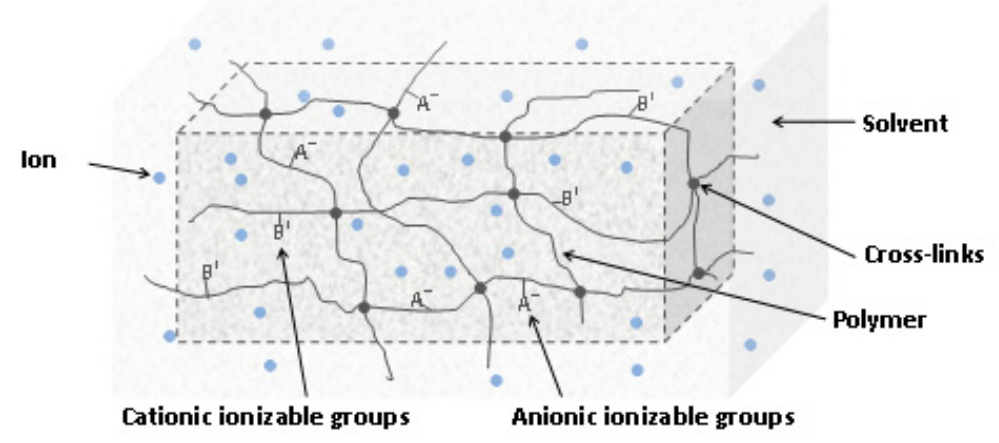

Figure 1. A schematic presentation of a gel immersed in a solvent. 
Networks of cross-linked polymers (hydrogels) exhibit both liquid-like and solid-like behavior [1-4]. Polymer networks are able to absorb water up to a thousand-fold of the dry weight of a polymer [5]. The unique property of hydrogels is the abrupt volume changes under transition from their collapsed to swollen state in response to external stimuli [6-14], see Figure 2. Due to this property, hydrogel is an "intelligent" material suitable for design of regulated systems. Hydrogels are especially desirable for bioanalytical applications because of their high water content and elastic nature similar to natural tissue.

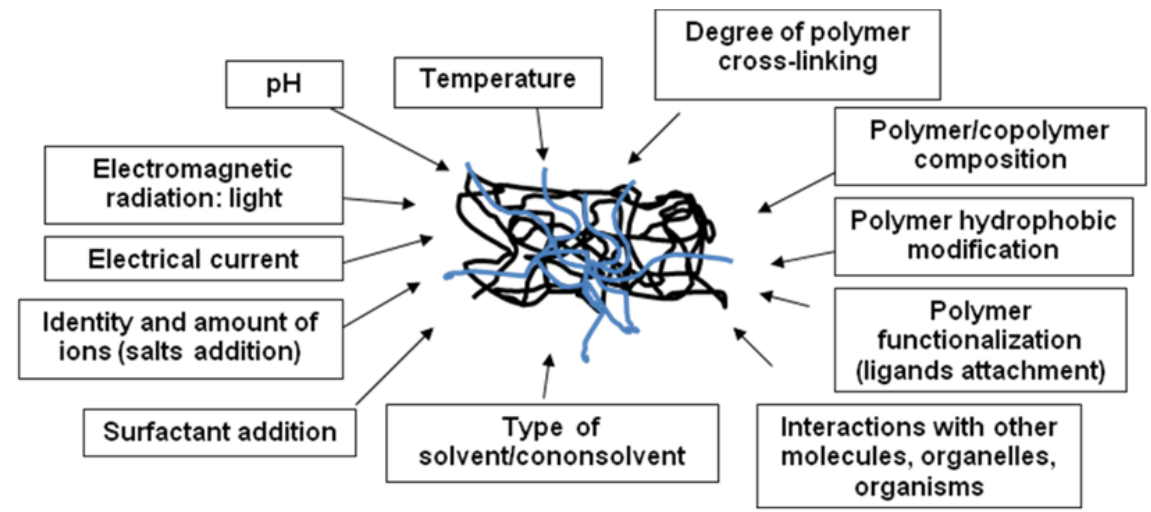

Figure 2. Different effectors of the swelling capacity of polymer networks (hydrogels).

Thermodynamically, the ability of hydrogels to swell in the course of water absorption is the matter of solubility of macromolecules comprising their 3D networks. We have already described elsewhere [15] possible types of solubility phase diagrams available within a window of the experimentally accessible thermodynamic parameters (e.g. temperature, pressure, concentration).

Various types of hydrogels based on both natural (e.g., hyaluronic acid, collagen, chondroitin sulfate, alginates, fibrin, and chitosan) and synthetic polymers made of neutral (e.g., 2hydroxyethyl methacrylate, N-alkylmethacrylamides, N-alkylacylamides, N,Ndialkylacrylamides), acidic (e.g., acrylic acid, metacrylic acid, 2-acrylamido-2-methyl propane sulfonic acid), and basic (e.g., N,N-dialkylaminoethyl methacrylate, 1-vinylimidasole, methacryloyoloxyethyltrialkylammonium bromide) monomers have been prepared, studied, and used in numerous applications (bioseparation, tissue engineering, sensing and molecular recognition, drug and gene delivery, controlled release, artificial muscles, flow control). It is not our intention to present a comprehensive review on hydrogel properties and applications. In this section, we would like to let the readers get a feeling of how significant the volume of hydrogel matrix can change in response to different stimuli.

\subsection{Effectors of volume change in hydrogels}

Temperature. The most pronounced volume changes of hydrogels are observed at the volume phase transition corresponding to the solubility phase diagram of a polymer in the hydrogel 
network. Many polymers exhibit coexistence curves with LCSP, so that the range of transition temperatures $\left(\mathrm{T}_{\mathrm{c}}\right)$ and concentrations are dependent of the chemical nature of the polymer. As a consequence, a temperature range where the hydrogel shrinks expelling water upon heating intrinsically depends on the type of polymer constituting its network. Figure 3 shows the examples of temperature dependencies of the swelling ratio for different polymeric hydrogels.

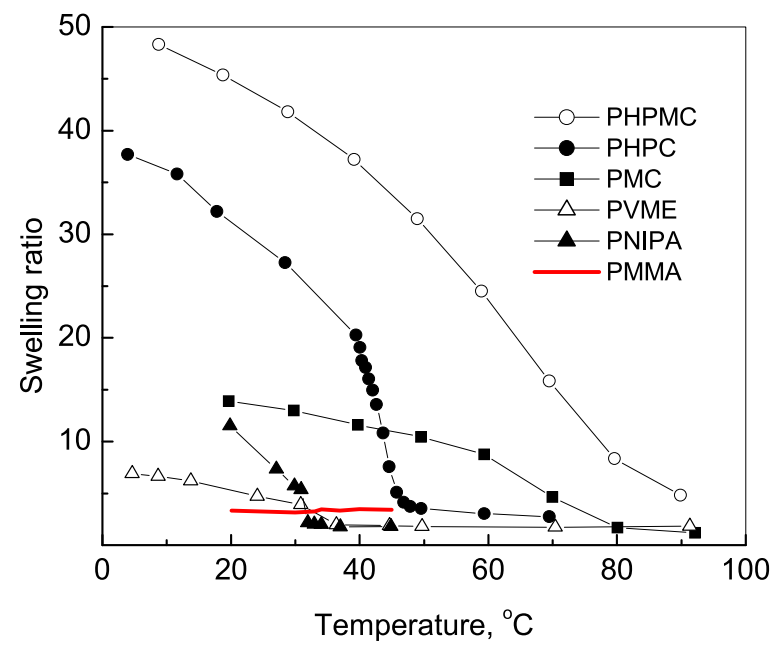

Figure 3. Relative volume changes as a function of temperature for hydrogels of different types: MC methylcellulose, HPMC - hydroxypropylmethylcellulose, HPC - hydroxypropylcellulose, NIPA - Nisopropylacrylamide, MMA - methacrylic acid, VME - vinyl methyl ether. The hydrogel transition temperature is close to the polymer LCST. PMAA hydrogel is not temperature sensitive because PMAA polymer is supposed to not exhibit a solubility phase transition at the experimentally achieved thermodynamic conditions. Data are adapted from [16-18].

The studies $[16,19,20]$ show that increased cross-linking may significantly decrease swelling ability of hydrogel, especially, below $\mathrm{T}_{c}$, but has a little effect on the value of $\mathrm{T}_{\mathrm{c}}$. These conclusions agree with our results (Figure 4).

It has been shown [21] that incorporation of a small amount of ionizable groups into the nonionic gel network drives the volume phase transition from continuous volume changes toward discontinuous one. Figure 5 explicitly demonstrates that an increase in the portion of sodium acrylate with carboxylic groups on the PNIPA network increases $T_{c}$ and extent of swelling ability below the transition temperature.

It is worthy to highlight, that the volume changes in a water-swollen hydrogel are expected to be continuous as a function of environmental stimuli, if the system remains totally miscible at given thermodynamic conditions. On the contrary, if changes in chemical nature of the polymer network, solvent quality, or environmental stimulus "push" the system into a two-phase (unstable) region of the solubility phase diagram, a swollen hydrogel collapses to a shrunken state due to reduction in solubility. During such volume phase transition, one can expect that properties of the hydrogel, most notably its volume, change discontinuously. 


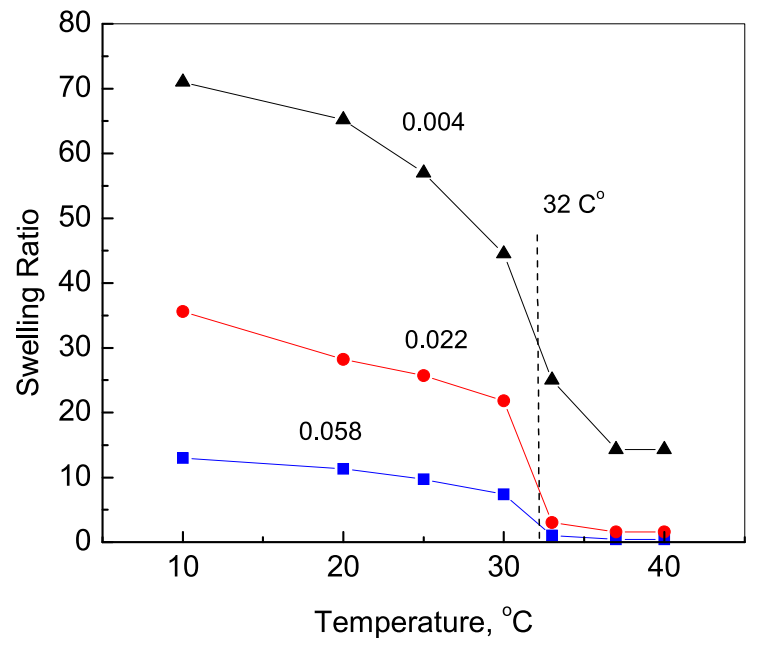

Figure 4. Swelling ratio $\left[\mathrm{Q}=\left(\mathrm{W}-\mathrm{W}_{0}\right) / \mathrm{W}_{0}, \mathrm{~W}_{0}\right.$ is the weight of dry gel $]$ of PNIPA hydrogel cylinders $(\varnothing 10$ $\mathrm{mm} \times 10 \mathrm{~mm}$ ) as function of temperature for different relative amount of the cross-linker (methylenebisacylamide, MBA) in the hydrogel-forming solution. The numbers near the curves are the values of ratio: $\mathrm{n}_{M B A} /\left(\mathrm{n}_{M B A}+n_{N I P A}\right)$, where $\mathrm{n}_{M B A}$ and $\mathrm{n}_{N I P A}$ are the number of moles of MBA and $N$ isopropylacrylamide (NIPA), respectively, at constant $\mathrm{n}_{\mathrm{MBA}}+\mathrm{n}_{\mathrm{NIPA}} \approx 0.66$.

Ions and ionic strength. Figure 5 also suggests that incorporation of charged (anionic, cationic, or both) groups on the polymer network makes the volume transition temperature and degree of swelling dependent on $\mathrm{pH}$ and ionic strength. Indeed, the poly( $N$-isopropylacrylamide-co-methacrylic acid) (PNIPA-MAA) microgel particles [22] at $\mathrm{pH} 3.4$ exhibited a decrease in $\mathrm{T}_{\mathrm{c}}$ from 33.5 to $28 \mathrm{C}^{\circ}$ with an increase in MAA content, whereas at $\mathrm{pH} 7.5$, the higher MAA content resulted in the higher $\mathrm{T}_{\mathrm{c}}$. In weakly charged PNIPA hydrogels, addition of ionizable groups on the polymer network pronounced the volume changes when temperature crossed $T_{c}[21,23,24]$. The experimental studies [25] revealed that even distribution of ionic groups in the network affects the temperature of volume change transition.

The type of ionizable groups on the polymer networks makes the maximum swelling ability of a gel strongly dependent on $\mathrm{pH}$. For example, the anioic PNIPA-MAA microgels exhibited the maximum swelling in the range of pHs from 6.5 to 10 [22 ], whereas for the cationic PNIPA-VI nanogels (VI stands for 1-vinylimidazole) the maximum swelling ratio was observed in the range of pHs from 6 to 3.5 [26]. It becomes even more intriguing if the so-called polyampholyte hydrogels are designed [27] by addition of both cationic (VI) and anionic (AA) groups on the network. The polyampholyte gel was in a shrunken state in the vicinity of the isoelectric point $(\mathrm{pH} \sim \mathrm{pI})$, and it swelled at both higher and lower pHs. It is interesting that such designed polyampholyte gels can work like biochemo-mechanical systems in which the enzymatically induced $\mathrm{pH}$ changes control the volume of polyampholyte network or, in opposite direction, the $\mathrm{pH}$ sensitive volume changes control the activity of enzymes immobilized into the gel [28]. 


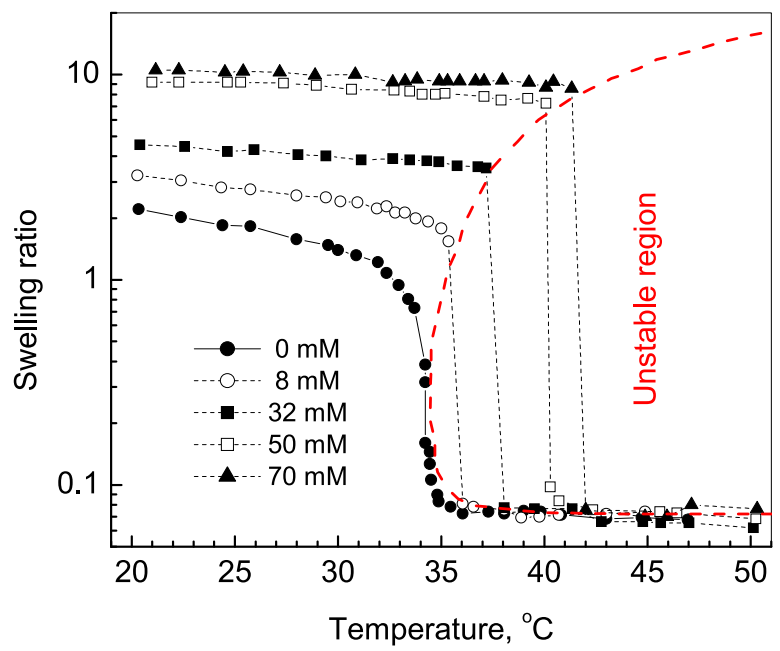

Figure 5. The degree of swelling of the poly(N-isopropylacrylamade-co-sodium acrylate) gel in water as a function of temperature. Numbers are the molar concentrations of sodium acrylate in the preparations. Data are adapted from [21].

There are experimental evidences [29-31] of de-swelling effects of different monovalent $\left(\mathrm{Li}^{+}\right.$, $\left.\mathrm{Na}^{+}, \mathrm{K}^{+}, \mathrm{Cs}^{+}\right)$and divalent $\left(\mathrm{Ca}^{2+}, \mathrm{Mg}^{2+}, \mathrm{Sr}^{2+}, \mathrm{Ba}^{2}\right)$ ions in hydrogels of different chemical nature, including the biologically relevant gels [32] and cross-linked DNA [33]. Interestingly, at the same molar ratios of divalent to monovalent cations, $\sim 1 \mathrm{mM}$ to $30 \mathrm{mM}$, respectively, similar volume changes were observed in biological polyelectrolyte systems during physiological processes like nerve excitation, musle contraction, and cell locomotion [34-40].

Surfactants. The extensive theoretical [41-43] and experimental [44-48] studies have shown that addition of anionic, cationic, and nonionic surfactants to the solution containing a gel can also influence the volume phase transition temperature and swelling degree of hydrogels depending on their hydrophobicity and charge of the polymer network. In general, addition of anionic or cationic surfactant to the solution of nonionic hydrogel rises the transition temperature as well as the swelling range, whereas the nonionic surfactant does not affect the transition temperature or the volume change. The surfactants with ionic head groups convert the neutral hydrogels to a polyelectrolyte gels when bind to the nonionic polymer networks, so that the transition temperatures elevate due to introduction of additional osmotic pressure by ionization. The changes in the volume phase transition are also dependent on the length of hydrophobic tail of ionic surfactants and the critical concentration of micelle formation. Indeed, it was found [45] that the transition temperature of nonionic poly(acryloyl-L-proline methyl ester) hydrogels increases more drastically in solutions of anionic sodium alkane sulfonate surfactants with the higher number of methylene units in the tail and at lower concentration than those with the shorter tails. The other interesting findings are [47]: (i) the amount of an ionic surfactant bound onto the swollen network of the nonionic PNIPA hydrogel is much greater than that to the collapsed 
one, (ii) on the contrary, the amount of nonionic surfactant bound onto the collapsed network of the PNIPA gel is greater than that on the swollen one, (iii) the transition temperature changes with the amount of the surfactant bound much stronger for anionic surfactants (e.g., sodium dodecyl sulfonate) than for cationic ones (e.g., dodecylamine hydrochloride), (iv) the distribution of anionic surfactant (e.g. sodium dodecylbenzene sulfate) bound within the PNIPA hydrogel is appeared to be heterogeneous [48] - the surfactant concentration is higher in the vicinity of the gel surface, whereas a central region of the gel may not contain any bound surfactant molecules. Therefore, there can be a gradient of swelling ability along the depth of surfactant penetration into the gel, namely: the peripheral layers are in the more swollen state with a higher transition temperature in comparison with the hydrogel core.

The uptake of a surfactant into the ionic and neutral polymer networks has been found to be different [49,50]. Particularly, when the cationic surfactant (e.g., $\mathrm{N}$-alkylpyridinium chloride, $\left.\mathrm{C}_{\mathrm{n}} \mathrm{PyCl}, \mathrm{n}=4,8,10,12,16\right)$ was absorbed from the solution into the anionic hydrogel [e.g., poly(sodium 2-acylamido-2-methylpropane sulfonate) (PNaAMS) gel], the polymer network was collapsed not swelled, herein the degree of shrinking increased with the length of the surfactant alkyl tail. Surprisingly, the surfactant influx $\left(\mathrm{mol} / \mathrm{s} / \mathrm{cm}^{2}\right)$ was enhanced by the increase in the network density. It was also observed that ionic strength significantly affects the uptake of surfactants into PNaAMS hydrogel, namely: (i) in pure water, the hydrogel began absorbing surfactants at concentrations below $10 \mu \mathrm{M}$ and the level of fluxes was high for alkyl tails of different lengths, (ii) in the $10 \mathrm{mM} \mathrm{NaCl}$ solution, the uptake of surfactants was practically absent at the concentrations lower than $100 \mu \mathrm{M}$ of $\mathrm{C}_{12} \mathrm{PyCl}$ and $400 \mu \mathrm{M}$ of $\mathrm{C}_{10} \mathrm{PyCl}$, but abruptly increased at higher concentrations, especially for surfactants with longer tail, e.g., the surfactant flux jumped $\sim 3$-fold for the surfactants with the number of carbon atoms in the alkyl tail greater than 10 . The kinetics of surfactant uptake clearly indicated [ 50 and references therein] that binding process significantly increases the amount of surfactant absorbed by the gel and slows down the diffusion of surfactant inside the gel. Theoretical and experimental analyses $[43,48-50]$ also showed that the closer to the surface of the gel, the higher is the concentration of the bound surfactant. Herein, this nonuniformity of surfactant binding increases with the cooperativity of binding in the presence of a salt. Thus, it was predicted that the progress of surfactant binding within the gel can be observed as the movement of the front of network collapse from the surface toward the center of gel.

Light. Hydrogels sensitive to light have been reported as well. For example, photosensitive gels with incorporated photosensitive molecules, such as leucocyanide and leucohydroxide, into the gel network have been reported [51]. These gels underwent volume changes upon irradiation and removal of ultraviolet light as a result of ionization reaction and internal osmotic pressure initiated by UV light. Significant volume changes in hydrogels can be also induced by visible light [52]. The mechanism of volume transition is different; it is due to direct heating of the polymer network by light. By the way, the fact that this process is extremely fast is of great importance from the practical point of view. 
More recent reports [53] show that a focused laser beam was able to generate the radiation force which induces reversible shrinkage in polymer gels. Control experiments confirmed that the laser-induced volume phase transitions are due to radiation forces, rather than local heating, modifying the weak interactions in the gels. Due to shear-relaxation processes, gel shrinkage occurs up to several tens of micrometers away from the irradiation spot. The compaction was also found in acrylamide gels, which are not temperature sensitive. In hydrogels with temperature sensitive volume phase transitions, such as PNIPA gel, it was found that the radiation force of the laser beam not only induces the volume phase transition, but also lowers the transition temperature $\mathrm{T}_{\text {tr }}$ by about $10{ }^{\circ} \mathrm{C}$ at an irradiation power of $1.2 \mathrm{~W}(\lambda=1064 \mathrm{~nm})$.

The light induced volume changes in photosensitive hydrogels have received increasing attention because of their scientific and technological significance for the so-called shapememory polymeric systems [54]. Being exposed to the light with lower wavelengths, the shape-memory materials become deformed and their temporary shape is fixed due to crosslinking. When irradiated with higher wavelengths, they recover their initial shape because of cleavage the cross-links.

Electrical field. Back in 1950s, it was found that contraction, oscillation, and bending of polyelectrolyte gels can be activated electrically [55-57]. In particular, gels prepared from polymers and copolymers that contain ionizable groups exhibited remarkable contraction when placed between a pair of electrodes connected to a direct current source. Polymer gels containing no ionizable groups showed no volume change under electrical field applied. The extent and rate of volume change of the polyelectrolyte gels were shown to increase with increasing electrical field [58]. An increase in the ionic strength (e.g., an addition of $\mathrm{NaCl}$ ) also increases the rate of gel shrinkage, whereas an addition of organic solvent (e.g., acetone or ethanol) decreases both the extent and the rate of shrinking. In different types of hydrogels, an electrically activated volume changes were associated with the induction of the medium $\mathrm{pH}$ change by the electric field [59], the electrically initiated volume phase transition [60], and the so-called electrokinetic phenomena - ion transport of counter-ions in the electric field [61].

The reversible shrinking and swelling of the polyelectrolyte gels stimulated by an electric field have become a basis for development of the so-called mechanochemical (chemomechanical) devices (engines) [62] functioning as actuators and artificial muscles [6365] and drug delivery systems [66].

\subsection{Supported hydrogel films}

Polymer networks being capable of stimuli responsive volume changing and controllable mass transferring have been increasingly recognized as building blocks in microfluidic devices (sensors, actuators, fluid pumps, and valves) [67], scaffolds in tissue engineering and regenerative medicine [68-70], actuators in artificial muscle development [63-65], and targeted and controlled release elements in drug delivery systems [17], including iontophoresis [71]. Hydrogels are flexible and fragile and require mechanical support. The 
idea of a supported hydrogel has been employed for decades in the planar configurations. Deposition of planar polymer networks onto supports allows for

i. biofunctionalization of surfaces, providing a natural environment for immobilization of highly specialized proteins, lipids, carbohydrates, and nucleotides

ii. preparation of flat homogeneous surfaces with nanoscale smoothness and heterogeneous substrates with more complex structures

iii. development of "cushions" that effectively separate from the solid surface more fragile layers, such as lipid bilayer membranes with reconstituted proteins

iv. detection of the changes in electrical or optical signals induced by the events of binding/dissociation or variations in the integral characteristics (hydrophobicity, charge, etc.) in relation to varied degrees of swelling

v. miniaturization of the abovementioned devices and systems.

\subsection{Methods for planar hydrogel micropatterning}

In the development of the intelligent biomaterials and biochips, based on the supported polymer networks, one goal is to synthesize regions with different physico-chemical properties: e.g., hydrophobic/hydrophilic, a wide range of shapes, thicknesses and dimensions, sensitive to different stimuli ( $\mathrm{pH}$, temperature, electrical field, radiation, etc.). Coupled with the need to synthesize regions of different properties, there is also a need in techniques to form high aspect ratio structures. Optical lithography or photolithography is the most suitable technique for micropatterning cross-linked polymer networks as high aspect ratio structures with features of controlled surfaces. The change in the solubility of polymer-coated substrate after exposure to radiation allows the selective removal of one part of the polymer coating, if irradiated part becomes soluble in the developer, it is called a positive-tone resist. When it becomes insoluble, it is called a negative-tone resist. The latter one provides a tool for preparation of patterned hydrogels, when water-soluble polymer subjected to the cross-linking step [72]. State-of-the-art photolithographic techniques are capable of mass-producing patterned structures in thin films with feature sizes as small as $\sim 250 \mathrm{~nm}$ with possible extension to features as small as $\sim 100 \mathrm{~nm}$ by use of a combination of deep UV light and improved photoresists [73]. However, the so-called $100 \mathrm{~nm}$ barrier cannot be easily surmounted. Advanced lithographic techniques, such as extreme UV (EUV) lithography, soft X-ray lithography, e-beam writing, focused ion beam (FIB) writing, and proximal-probe lithography $(74,75)$, have the capability of generating extremely small features (as small as a few $\mathrm{nm}$ ), but their development for manufacturing of nanostructures requires substantial effort and they, as the conventional photolithography, are poorly suited for patterning non-planar surfaces.

\subsection{Evanescent wave as a tool for nanoscaling}

To improve the resolution, a procedure for the fabrication of very thin nanoparts has been proposed - photopolymerization by evanescent waves (PEW) [76]. 
The total internal reflection (TIR) is the phenomenon guiding light in a higher refractive index (RI) medium [77]. Under conditions of TIR, light can totally reflect from a dielectric interface, remaining inside the medium of a higher RI ( $\left.\mathrm{n}_{1}\right)$. The critical angle $\left(\theta_{c}\right)$, at which the reflected ray will travel along the boundary surface, is determined by the ratio $\mathrm{n}_{2} / \mathrm{n}_{1}$, where $\mathrm{n}_{2}$ is the lower RI of the second medium:

$$
\theta_{c}=\arcsin \left(n_{2} / n_{1}\right)
$$

During the propagation of electromagnetic radiation inside high RI medium, a portion of the electric field can penetrate outside, to the medium of the lower RI. The penetrated electric field is called the evanescent wave. The intensity of the evanescent wave decays exponentially [77] as follows:

$$
E(z)=E_{0} \exp (-z / d),
$$

where $\mathrm{E}_{0}$ is the amplitude at the interface and $\mathrm{z}$ is the distance outside the high RI medium. The depth of penetration of the evanescent wave is the distance at which the intensity of the electric field has decayed to $1 / \mathrm{e}$ of that at the interface:

$$
d=\frac{\lambda}{2 \pi n_{1} \sqrt{\sin ^{2} \theta-\left(n_{2} / n_{1}\right)^{2}}},
$$

where $\theta$ is the incident angle and $\lambda$ is the wavelength of the radiation. The penetration depth of the evanescent field can be interpreted as the thickness of the layer in which acting light is confined. Typically $d$ is about few tenth of $\lambda$. Within the depth of penetration, the evanescent wave can interact with molecules located nearby the interface. Thus far, the evanescent wave can be absorbed within the polymerizable formulation to initiate a polymerization reaction around the area of the interface exposed to the light (Figure 6).

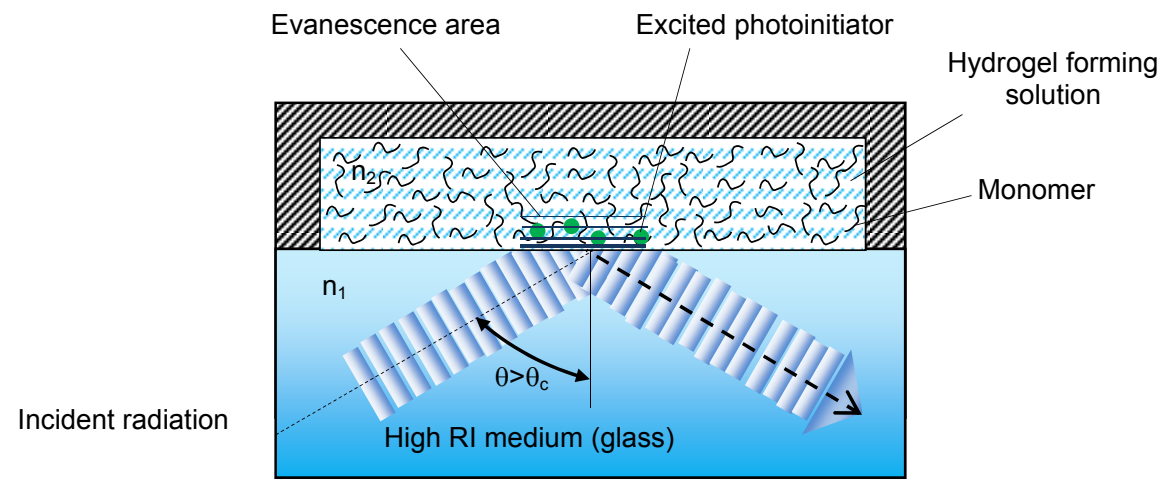

Figure 6. Schematic representation of photopolymerization by evanescent waves in the planar configuration. The laser beam is totally reflected at the interface between the high refractive index glass and the hydrogel forming solution, as the angle of incidence is greater than a critical angle $\theta_{c}$. Close to the interface, the electromagnetic field is non-zero in the polymerizable medium but decays exponentially to zero. 
It has been shown [76] that the thickness and the shape of the hydrogel planar films obtained by this lithographic technique correlated with the properties of the incident radiation. In particular, a linear dependence of the film thickness on the logarithm of the exposure time was observed. The film thickness varied with the angle of incidence of the laser beam at the surface of the photopolymerizable mixture. When the wavelength of laser used was $514 \mathrm{~nm}$, the films of $30 \mathrm{~nm}$ thickness were obtained. To fabricate homogeneous planar films, the light intensity had to be constant over the whole irradiated area. On the other hand, it was demonstrated that 2D and 3D photolithography were also possible, i.e. when the intensity of the incident radiation was not uniform at the interface, the polymerized parts were non-planar. The light intensity at the interface was modulated using photomasks or causing interfering pattern in the evanescence plane. It was also possible to control the thickness of the hydrogel layer locally by varying the exposure time. A diaphragm of variable diameter was imaged at the glass/solution interface, so that the central part was illuminated for a longer time than the peripheral area - a stepwise structure with controllable height of every step was obtained. These experiments present the indications that PEW may provide a simple method of stereophotolithography well suited for patterning non-planar surfaces.

\subsection{Hydrogel/optical fiber assembly}

Cylindrical surface of optical fiber core seems to be the next step in the development of methods for micropatterning non-planar supporting surfaces. Cylindrical surfaces of optical fiber core offer several advantages over conventional planar configuration, e.g.:

- Optical fibers provide the method for monitoring optical signal of different spectral composition

- They have potential for a higher density of extremely small features (as small as a few $\mathrm{nm}$ ) deposited on the cylindrical surface

- Regions with different physico-chemical properties can be synthesized and multiple reagents can be immobilized on their surfaces

- Simultaneous response to more than one analyte can be recorded, stored, and interpreted

- They allow devising the miniature devices which can be used for monitoring parameters in vivo

The idea of a hydrogel/optical fiber assembly has been already employed for decades mostly in the context of the fiber optic chemical and biochemical sensors [78-81]. The features of hydrogels relevant to the purpose of optical fiber coating are: satisfactory light transmission, low refractive index (lower than that of glass), compatibility with glass substrates, permeability for water-born analytes, and mechanical stability due to cross-linking. The work on sensors development has also shown that cross-linked polymer matrix on the optical fiber is of great potential due to its functionality:

1. hydrogel capable of swelling and shrinking can produce microbending in fiber [82],

2. it can absorb the evanescent tail of the light propagating within the fiber core $[83,84]$, and 
3. hydrogel matrix can serve as a container for ligands, which are normally adsorbed onto [85], entrapped into [86-88] or covalently bound to [89-91] the supporting matrices, so that biological recognition processes through antigen-antibody, enzyme-substrate, or ligandreceptor interactions can be incorporated to identify and quantify biochemical substances.

There exist numerous sensing schemes [78-81], however, a simple analysis shows that only two methods of deposition of hydrogel matrix on an optical fiber have been employed: (i) hydrogel spots were polymerized on fiber distal ends (not on the side wall of a fiber core) by direct light $[78,92,93]$ and (ii) hydrogel was deployed on side-surface of a fiber core by spreading a preliminary prepared polymer followed by its further cross-linking [94].

The disadvantage of the polymer matrices deposited by those "macroscopic" methods is in that they are relatively thick (microns to $\mathrm{cm}$ ). As a result, the polymer matrix exhibits a prolonged response time (minutes to days), hampered expansion and contraction, and hindered mass transfer. Thus, it is advantageous to have a cross-linked polymer matrix of nanometer thickness (hydrogel nanofilm) as a prerequisite for future applications. The bottom up question is how to prepare the nanometer sized hydrogel layers with controllable thickness. Polymerization by evanescent waves (PEW) has been shown [76] to be a versatile and convenient method for fabrication of nano- and microparts with controllable submicrometer heights on the planar surface of glass slide. We have proposed the employment of evanescent waves for preparation of ultrathin hydrogel films in the proximity of the side surface of a fiber core, i.e. in cylindrical configuration [95]. We believe that cylindrical surface of an optical fiber core is more practical compared to a flat surface to support ultrathin cross-linked polymer fabrics. First, the cylindrical architecture of the "nanofilm/fiber structure" will not allow the polymer "cylindrical sock" to come out of the fiber core. Second, optical fiber connected to a spectrometer is a polymerization setup and an analytical device in one. Considered below are the distinct prospects of using the combination of a spectroscopic tool (optical fiber) and environmentally responsive element (cross-linked polymer matrix on side wall of optical fiber core) in various bioanalytical applications. The cylindrical configuration of the hydrogel/fiber structure is a promising approach to miniaturization of lab-on-a-chip devices.

\section{Basic model of polymerization by evanescent waves}

\subsection{Evanescent waves produced by TIR}

The evanescent waves have been widely used for the spectroscopic detection of chemical and biological species by fiber-optic sensors [78-81]. In our previous work [95], evanescent waves were used to initiate a polymerization reaction around a fiber core. This procedure is really new: (i) for the first time, EW were used to fabricate very thin polymer films of cylindrical configuration, (ii) for the first time, the PEW procedure is developed for a photopolymerizable formulation sensitive to ultraviolet light which is preferred for a higher spatial resolution, (iii) it requires no preforming of the polymer layer, (v) the thickness is just governed by the penetration of acting light into the polymerizable formulation, which in turn is a function of the radiation properties (intensity, exposure time, spectral composition), optical properties of 
fiber core and hydrogel forming solution (refractive indexes), and geometry of the fiber core (diameter). In other words, because of the propagation mode of EW produced by TIR, the PEW procedure is a unique way to reduce the interaction volume of light with the reactive formulation to the utmost, i.e. down to the nanometer scale - it looks possible to obtain cylindrical cross-linked polymer films with the record thickness of 1 to $5 \mathrm{~nm}$.

Consider a light beam that propagates in a higher index medium (refractive index $n_{1}$ ) of the fiber core and reflects at the interface with a lower index medium (refractive index $n_{2}$ ) of the hydrogel forming solution (Figure 7). The beam propagates by a series of total internal reflections at the core/solution interface. The interference between the incident and the reflected waves gives rise to standing waves inside the fiber core, perpendicular to the interface, and to the generation of an evanescent field inside the solution. The intensity of the electromagnetic field transmitted by EW decreases exponentially as it propagates farther away into the sample and is expressed as follows:

$$
I(z)=I_{0} \exp (-2 z / d)
$$

with $d$ expressed by Eq (3) has the dimension of a length and is called the characteristic penetration depth.

The evanescent wave can interact with molecules located within the depth of penetration. Thus far, the evanescent wave can be absorbed, scattered or can excite molecules around the area of a fiber core exposed for polymerization (Figure 7).

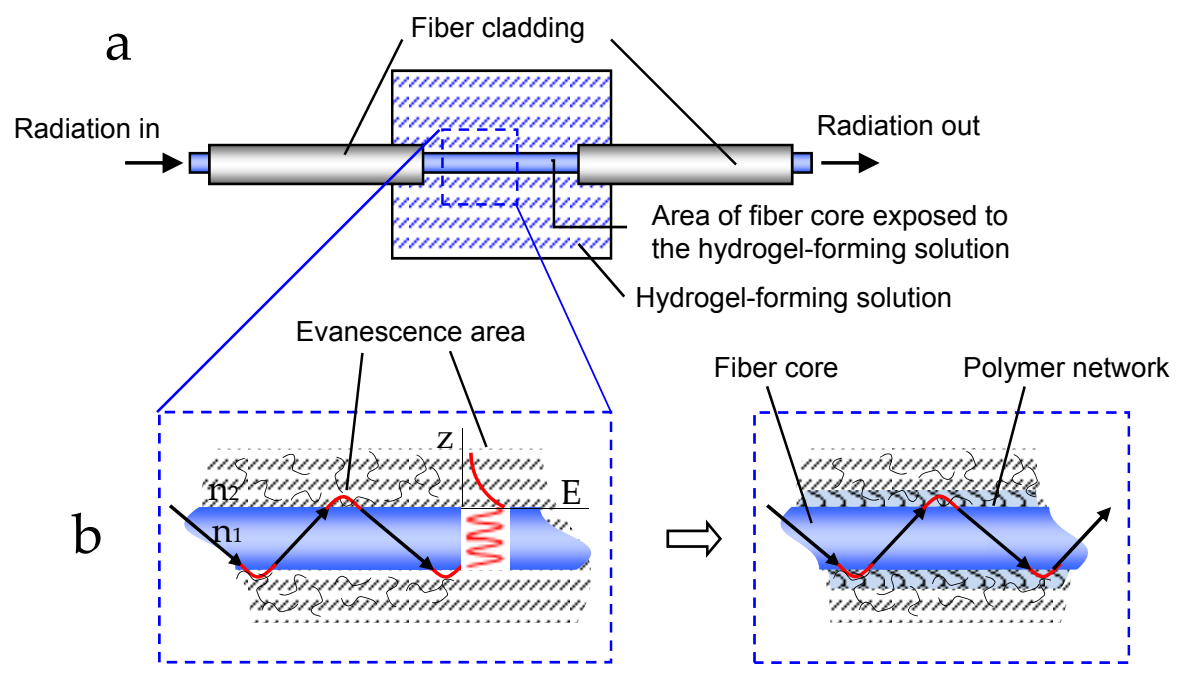

Figure 7. Optical fiber arrangement for polymerization by evanescent waves: (a) free-cladding area of fiber core exposed to the hydrogel-forming solution (cylindrical configuration); (b) in the course of the propagation of electromagnetic radiation inside the optical fiber, a portion of the electric field, which penetrates out of the fiber core (evanescent wave) initiates the polymerization reaction in nanometer layer around the fiber core. 


\subsection{Polymerization process}

Radical initiation of the photopolymerization generally provides high polymerization yield, and photosensitivity for radical polymerization can be extended in the visible range by adding a suitable sensitizer [96]. The radical photopolymerization requires three essential elements: electromagnetic radiation (hv), photoinitiators (PI), and mono/macromers (M) with unsaturated moieties such as a vinyl group and its derivatives. Ultraviolet $(190-400$ $\mathrm{nm})$ and visible light $(400-800 \mathrm{~nm})$ can be harnessed for photopolymerization. Accordingly, the maximal absorption wavelength $\left(\lambda_{\max }\right)$ of the photoinitiator is in the relatively high energetic UV or near UV visible range.

\subsubsection{Photopolymerization reaction scheme}

The photopolymerization of a monomer (M) in the presence of a photoinitiator (PI) can be represented schematically by the following equations:

Step 1. Radical formation and initiation of the polymerization

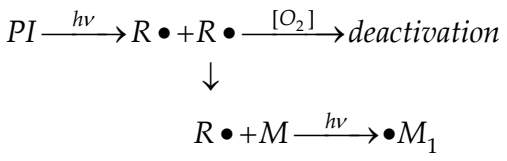

It is known [76] that in the early stages of irradiation, the absorbed light is used mainly to consume oxygen, which acts as a strong deactivating agent by trapping the formed radicals through peroxidation. This phenomenon is one of the main reasons accounting for a delay between the beginning of the irradiation and the start of polymerization. After a while, as the concentration of oxygen decreases, it becomes low enough and the reaction of initiating radicals with monomers competes successfully with the deactivation processes. The dose of energy absorbed before polymerization occurs, the so-called the threshold dose $E_{t h}$, is the dose of energy necessary to consume a sufficient amount of oxygen. By the way, polymerization by evanescent waves allows one to circumvent the problem: the total internal reflection provides the thinnest diffusion layer with the highest rate of oxygen consumption. Moreover, polymerization in $\mathrm{N}_{2}$ atmosphere (nitrogen-blanketing) is a precaution that allows substantial overcoming $\mathrm{O}_{2}$ inhibition of photopolymerization [98]. Therefore, assuming that the formation of monomer radicals $\bullet M$ is much faster process than the formation of radicals $R \bullet$, the initiation of polymerization in the absence of $\mathrm{O}_{2}$ is the ratelimiting process determined by the rate of $R \bullet$ formation:

$$
r_{I}=k_{I}[P I]
$$

where $P I$ is the concentracion of a photoinitiator, $k_{I}$ is the rate constant of the initial step. As monomer radicals $\bullet M$ are produced, the absorbed energy is used to form the $3 \mathrm{D}$ polymer network. 
Step 2. Propagation

$$
\begin{aligned}
& M+\bullet M_{1} \stackrel{h v}{\longrightarrow} \bullet M_{2} \\
& M+\bullet M_{2} \stackrel{h v}{\longrightarrow} \bullet M_{3} \\
& ------------ \\
& M+\bullet M_{n-1} \stackrel{h v}{\longrightarrow} \bullet M_{n}
\end{aligned}
$$

Polymer chains start growing and the cross-linking reaction proceeds rapidly to reach the gel point and finally vitrify. Letting $[\bullet M]$ represent the sum of concentrations of all radicals including $\bullet M_{i}$ and $R \bullet$, the rate of propagation determines the rate of polymerization:

$$
r_{P}=k_{P}[M][\bullet M],
$$

where $\mathrm{kP}$ is the rate constant of the propagation step. Mechanical resistance appears only when the dose of energy received by the system is greater than $E_{g e l}$.

Step 3. Termination

$$
\bullet M_{n}+\bullet M_{m} \rightarrow \begin{cases}M_{n+m} & \text { mutual termination } \\ M_{n}+M_{m} & \text { disproportionation }\end{cases}
$$

The termination of chain propagation usually proceeds by standard radical coupling (mutual termination), when two growing radical chains combine, or disproportionation, when unsaturation exchanges through $\beta$-hydrogen migration involving ambient oxygen [97]. The rate of termination can be expressed as follows:

$$
r_{T}=k_{T}[\bullet M]^{2},
$$

where $k_{T}$ is the rate constant of the termination step.

Assuming that monomer radical is formed very quickly and its concentration is constant in the course of the chain reaction (steady-state approximation), one can show that in the case of termination by coupling, the average degree of polymerization $\left\langle p_{n}\right\rangle$ is a function of the concentrations of monomer $[M]$ and initiator $[P I]$, which can be controlled experimentally:

$$
\begin{aligned}
& {[\bullet M]=\left(\frac{k_{I}}{k_{T}}\right)^{1 / 2}[P I]^{1 / 2}} \\
& \left\langle p_{n}\right\rangle=2 \frac{\text { the rate of propagation }\left(r_{P}\right)}{\text { the rate of termination }\left(r_{T}\right)}=\frac{2 k_{P}}{\sqrt{k_{I} k_{T}}}[M][P I]^{-1 / 2}
\end{aligned}
$$

Therefore, kinetics of chain reaction controls the polymerization as follows:

- The slower the initiation of the chain (the lower [PI] and the smaller $k_{I}$ ), the higher is degree of polymerization 
- The higher the concentration of monomer $[M]$, the longer is the average length of polymer chain

- The higher the concentration of initiator [PI], the shorter is the average polymer chain

\subsubsection{Time dependence of photopolymerization}

In order to find the time course of the concentration of the polymer $\left[M_{n}\right]$, we begin with the kinetic equation for polymerization

$$
-\frac{d[M]}{d t}=k_{P} \sqrt{\frac{r_{I}}{k_{T}}}[M] .
$$

Separating variables and integrating with the assumption of no time dependence for all terms except the concentration of monomer $[M]$ gives

$$
[M]=[M]_{0} e^{-k_{p} \sqrt{\frac{r_{L}}{k_{T}}} t} .
$$

The concentration of the polymer $\left[M_{n}\right]$ can be obtained from the mass conservation law and Eq.(5) for the average degree of polymerization

$$
\begin{aligned}
& {[M]_{0}=[M]+\left[M_{n}\right]\left\langle p_{n}\right\rangle} \\
& {\left[M_{n}\right]=\frac{[M]_{0}-[M]}{\left\langle p_{n}\right\rangle}=\frac{\sqrt{r_{I} k_{T}}}{2 k_{P}}\left(1-e^{-k_{p} \sqrt{\frac{r_{I}}{k_{T}}}}\right)}
\end{aligned}
$$

Expanding the exponential function into a series in powers of $x=k_{p} \sqrt{\frac{r_{I}}{k_{T}}} t$ and limiting ourselves with only two terms $\left(e^{-x} \approx 1-x\right)$, the increase of polymer concentration with time can be estimated as follows:

$$
\left[M_{n}\right] \approx \frac{r_{I}}{2} t .
$$

\subsubsection{Photochemical and processing parameters and the point of gelation}

At the gel point, $\left[M_{n}\right]=\left[M_{n}\right]_{c}$, the critical concentration of the polymer represents a critical conversion point of a gel formation and corresponds to the dose of energy $\left(E_{g e l}\right)$ absorbed by the system during the time of exposure $t_{e}$. Our goal is to find the relation between $E_{g e l}$ and the photochemical and processing parameters. The initiation rate $r_{i}(\mathrm{~mol} / \mathrm{L} / \mathrm{s})$ is related to the photonic flux $I(z)$ (= amount of photons per surface area per second, Einstein $/ \mathrm{m}^{2} / \mathrm{s}=10^{-3} \mathrm{~m}$ $x \mathrm{~mol} / \mathrm{L} / \mathrm{s})[98,99]$

$$
r_{I}=\phi \varepsilon[P I] I(z),
$$


where $\phi$ is the quantum yield of the photoinitiator, $\varepsilon$ is the molar extinction coefficient $(\mathrm{L} / \mathrm{mol} / \mathrm{m})$, and $I(z)$ is the incident intensity of the electromagnetic field transmitted by EW at depth z. In the absence of a photoinitiator, the photonic flux, or intensity, at depth $z$ decreases exponentially according to Eq. (4). However, in the presence of the molecules of photoinitiator additional absorption should be taken into account by Beer's Law factor:

$$
I(z)=I_{0} e^{-2 z / d} e^{-\varepsilon \ln 10[P I] z}=I_{0} e^{-z / d_{e f f}},
$$

where $I_{0}$ is the incident intensity at the fiber core/solution interface $(z=0), d_{e f f}=\frac{d}{2+d \varepsilon^{\prime}[P I]}$ is the effective penetration depth of the evanescent waves, and $\varepsilon^{\prime}$ stands for $\varepsilon \ln 10$. It seems reasonable that this effective depth of penetration of the evanescent field into the hydrogel forming solution is inversely related to the extinction coefficient and concentration of photoinitiator. Now, in the first approximation, the dose of energy received at depth $z$ and time $t$ is given by the following relationship:

$$
E(z, t)=I_{0} t e^{-z / d_{\text {eff }}} .
$$

We use this equation to define the $E_{g e l}$ as the dose of energy (= amount of photons per surface area, Einstein $/ \mathrm{m}^{2}=10^{-3} \mathrm{~m} \times \mathrm{mol} / \mathrm{L}$ ) absorbed by the system to polymerize a layer with thickness $l_{p}$ at exposure time $t_{e}$, i.e.:

$$
E_{g e l}=E\left(l_{p}, t_{e}\right)=I_{0} t_{e} e^{-l_{p} / d_{e f f}} .
$$

Substitution of Eqs. (16) and (13) into Eq. (12) at $z=l_{p}, t=t_{e}$, and $\left[M_{n}\right]=\left[M_{n}\right]_{c}$ allows the following expression to be obtained for the critical dose of energy necessary to reach the gel point as a function of the concentration of photoinitiator

$$
E_{g e l}=\frac{2\left[M_{n}\right]_{c}}{\phi \varepsilon[P I]}
$$

Note that the less the concentration of the photoinitiator in the polymerization formulation, the higher dosage is necessary to polymerize a stable film on the fiber core. Combining Eqs. (17) and (16), one can predict the dependence of the thickness of polymerized hydrogel on the photochemical properties of the hydrogel forming solution ([PI], $[M])$ and processing condition $\left(\phi, \varepsilon, I_{0} \times t_{e}\right)$

$$
l_{p}=d_{e f f} \ln \frac{I_{0} \times t_{e}}{E_{g e l}}=\frac{d}{2+d \varepsilon^{\prime}[P I]} \ln \frac{I_{0} \times t_{e}}{2\left[M_{p}\right]_{c} / \phi \varepsilon}[P I]
$$

With all these assumptions in place, one can conclude that the presence of photoinitiator results in the decrease in the effective depth of penetration of evanescent light because of its absorption by the photoinitiator molecules. The second and main finding is that for a given concentration of photoinitiator $[P I]$, the thickness of polymerized film $l_{p}$ remains zero until 
the dose of energy received by the system Ioxte exceeds the critical value defined by the critical concentration of polymer $\left[M_{n}\right]_{c}$ corresponding to the point of gelation:

$$
I_{0} \times t_{e}>E_{g e l}=\frac{2\left[M_{p}\right]_{c}}{\phi \varepsilon[P I]}
$$

According to this model, if $I_{0} \times t_{e}>E_{g e l}$, the polymer thickness around the fiber core is expected to increase linearly with the logarithm of $I_{0} \times t_{e}$, whereas the slope of this dependence is equal to the effective penetration depth of the evanescent wave.

\section{Optical fiber evanescent wave spectroscopy (FEWS)}

\subsection{Real time recording of polymerization on the side surface of optical fiber core}

The polymerization as well as observation and characterization of the nanofilm formation on the side surface of a fiber core, if any, are the-state-of-the-art and the "know-how". How to detect the formation of ultrathin films made of tiny cross-linked polymeric threads with optical properties similar to the solvent? This is the critical question. Experimental approaches to the question of hydrogel nanofilm monitoring usually fall into direct microscopic methods, such as optical microscopy (OM), atomic force microscopy (AFM), and electron microscopy (EM), which can be used at the step of film characterization. However, they are not convenient for routine measurements especially at the step of film formation. Spectroscopic methods are much practical in this case.

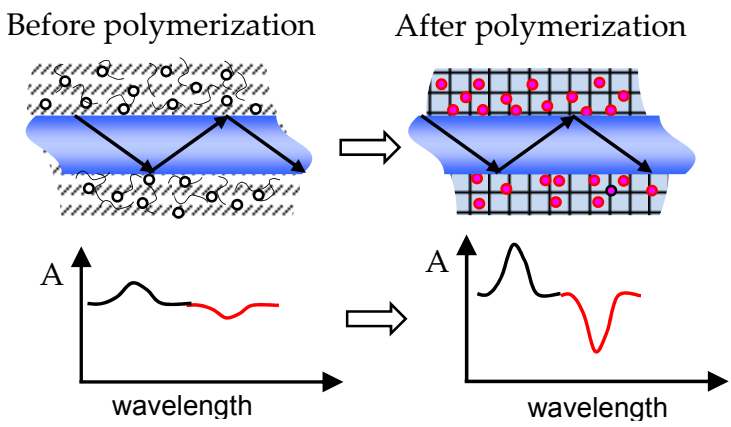

Figure 8. Spectroscopic detection of polymerization on the optical fiber core. The formation of polymer network changes the microenvironment of the fluorescent probe resulting in an increase in fluorescence emission (red line). Absorbance increases due to scattering on microheterogeneities of newly formed polymer network (black line).

Strikingly, the optical fiber arrangement for polymerization by evanescent waves (Figure 7) can be used for the real-time monitoring of hydrogel photopolymerization on the side surface of the fiber core. Indeed, in the course of initiation of polymerization, the intensity of light passing through the fiber core decreases due to excitation of molecules of 
photoinitiator, radical formation, and consumption of oxygen within the evanescence area. Furthermore, during polymerization the refractive index of the material changes, thus, inducing a modification of the refractivity of the core/solution interface. And finally, when the point of gelation is reached, the energy of evanescent wave scatters on the microheterogeneities of the cross-linked network.

The other way to record the polymerization on the side surface of a fiber core with time is to apply fluorescent probes. The fluorophores capable of changing the quantum yield of fluorescence depending on the microenvironment can be used (Figure 8). For example, 1anilinonaphthalene-8-silfonate (ASN) may exhibit 100-fold emission intensity in the polymerized network in comparison to the pre-polymerization solution. Moreover, the maxima of both absorption and fluorescence picks shift with changes in the polarity of the microenvironment [100,101].

\subsection{The ideas of hydrogel film detection by FEWS}

Actually, the measured polymerization kinetics itself is the evidence for hydrogel formation. Nevertheless, a hydrogel film, which has already been polymerized on the fiber core, could be detected spectroscopically. Indeed, studying the mechanical and electrochemical responses of hydrogels in the course of swelling/de-swelling of polymer matrix [102-106], we have observed that being swollen in water hydrogels were soft, gentle, and transparent materials, whereas under dehydration or wet/dry transition, polymer networks revealed a drastic volume decrease (up to 1000 times) resulted in the transparency drop. Thus, the correspondence between variations in physical properties (volume, thickness, density, refractive index, etc.) of hydrogel films and changes in the spectral composition and total intensity of light passing through the fiber stands behind the ideas of hydrogel film detection on the fiber core.

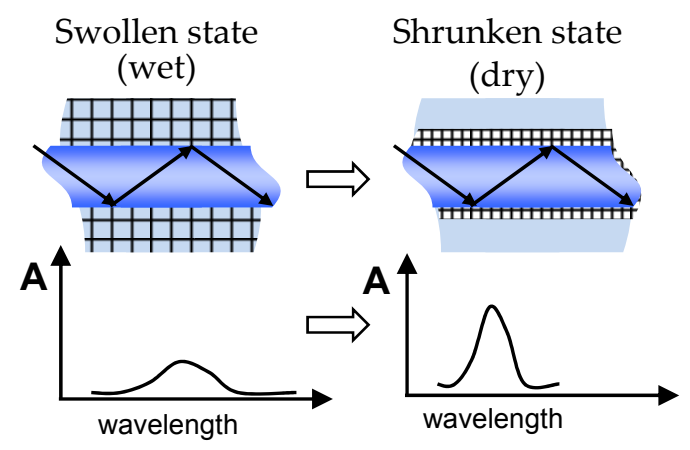

Figure 9. Spectroscopic detection of a polymer network on the optical fiber core: an increase in density (refractive index) of the polymer network in the course of dehydration of gel results in higher scattering/absorption of the evanescent light nearby the fiber core.

The first approach to the detection is illustrated in Figure 9. The scattering/absorption of evanescent light is greater in the dried polymer network than in its swollen wet state. 
The second approach is based on using fluorescent probes (e.g. fluoresceins) with a capability of self-quenching at high concentrations [107-109]. Fluorescence resonance energy transfer (FRET) is a distance-dependent interaction between the electronic excited states of two dye molecules in which excitation is transferred from a donor molecule to an acceptor molecule without emission of a photon. Fluorescein/fluorescein is a donor/acceptor pair with 4.4-nm Förster radius, the distance at which $~ 50 \%$ of excited donors are deactivated by FRET. Fluorescence intensity is expected to decrease in the course of nanofilm shrinkage (the distance between fluorescent molecules becomes smaller) and to increase with hydrogel swelling (Figure 10).

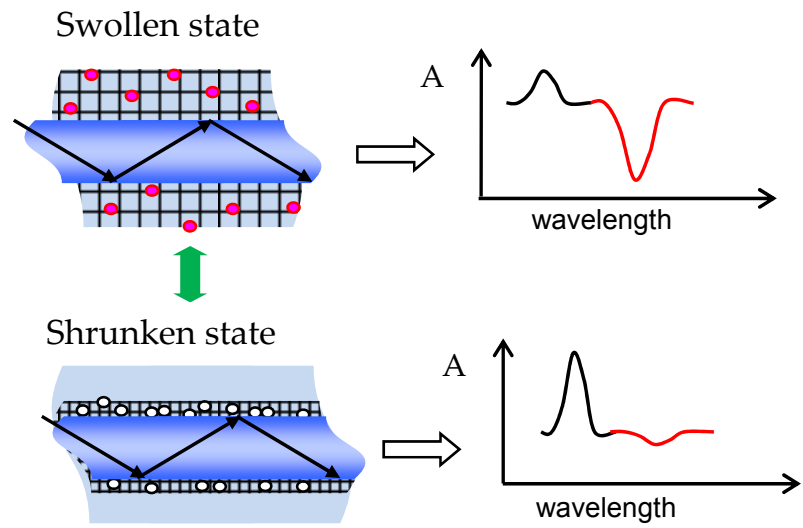

Figure 10. Spectroscopic detection of a polymer network on the optical fiber core: when fluorophores are loaded into the swollen hydrogel layer (red circles), the shrinkage of the polymer network will decrease the distance between these trapped molecules (white circles), resulting in a decrease in the fluorescence emission (red line). Herein, the absorption (black line) increases due to scattering of evanescent light on the denser polymer network.

\subsection{Cylindrical hydrogel film characterization by FEWS}

To the best of our knowledge, the first report on polymerization of hydrogel in a cylindrical configuration using the evanescent tail of light propagating in fiber was published in 2010 [95]. So long the hydrogel is on the fiber core, experimental techniques concerned with characterization of the extent of its volume change modality are in demand. The formed cylindrical polymer network is supported by, but not covalently bound to the side-surface of fiber core. It is a kind of "cylindrical sock" on a fiber core, and its thickness can vary with the photochemical and processing parameters discussed in Section 2.2.3. Understanding of the hydrogel functionality, specificity, and biochemical activity is required as well. It looks quite relevant to use the optical fiber evanescent wave spectroscopy (FEWS) as a method for characterization of hydrogels on the fiber core:

On the one hand, because hydrogels are the so-called soft matter materials with environmental response, i.e. relatively small external perturbations can cause dramatic 
structural changes in them followed by substantial physical expansion/contraction. Since the perturbed polymer networks with swelling/de-swelling ability in many cases, are not in equilibrium, it is of great importance to characterize the relaxation of their physical dimensions (especially, at the nanometer length scale) with time.

On the other hand, because the experimental scheme of FEWS allows one not only to record time-changes in integral intensity of light passing through the fiber, but also to provide a novel information on the spectral composition of light scattered/absorbed within the medium in the immediate vicinity of the fiber core surface, and how the spectrum changes with time at different external conditions, thereby taking advantage of time-resolved and nanometer-scaled measurements.

Thus, the hydrogel/fiber optics assemblage offers a method for studying the kinetics of swelling and shrinking spectroscopically with minor modifications of the same experimental scheme as used for polymerization and detection.

\section{Experimental procedures}

\subsection{Photopolymerizable formulations}

Even a brief review of physical and chemical properties of hydrogels present in Introduction allows the reader to realize that any hydrogels with relevant chemical composition and environmental responsiveness could be synthesized for numerous applications. The photopolymerizable formulations are specified by the desirable functionality and specificity of the hydrogel film on the fiber core. For example, in order to prepare a temperature sensitive hydrogel, $\mathrm{N}$-isopropylacrylamide (NIPA) monomer can be used, since it forms polymers with volume phase transition temperature varied from 20 to $45{ }^{\circ} \mathrm{C}$, i.e. in the physiologically relevant range [110]. Combined temperature and ionic sensitivity of the hydrogels can be achieved by co-polymerization of NIPA with corresponding cationic (e.g. vinylimidazole) or anionic (e.g. carboxyl) moieties [8-10]. Hydrogel materials with biological responsiveness built in can be achieved by introducing ligands that bind specific biomolecules into the the material matrix [111]. When exposed to a biological target (nutrient, growth factor, receptor, antibogy, enzyme, or whole cell), molecular recognition events in the bioresponsive hydrogels trigger macroscopic responses, such as swelling/deswelling or solubility transitions.

The cross-linking density, which is the main effector of swelling ability, can be varied by changing the [monomer]/[cross-linker] ratio. As a standard cross-linker $N_{,} N^{\prime}$ methylenebisacrylamide (MBA) is used in many formulations. To optimize the composition of polymerizable formulations in terms of the final swelling/de-swelling ability, a hydrogel forming solutions of different composition should be tested on bulk gels by varying concentrations of monomer, co-monomer, and cross-linker (see Figure 4).

To prove the concept of photopolymerization by evanescent waves on the side surface of the optical fiber core, the composition of temperature sensitive PNIPA hydrogel has been 
optimized. The method for preparation of a bulk PNIPA hydrogel by polymerization of a hydrogel-forming medium induced by a UV-lamp $(\sim 365 \mathrm{~nm}, 100 \mathrm{~W})$ has been described elsewhere [102,103]. From the homogeneity standpoint, it was found that during polymerization the temperature should be kept below the Lower Critical Solution Temperature (LCST, $\mathrm{T}_{\mathrm{c}}$ ) of the final hydrogel. Particularly, the polymerizable aqueous solution of NIPA (5 wt \%) and MBA (0.5 wt \%) yielded a clear temperature-responsive hydrogel with 10 -fold volume change in water near $\mathrm{T}_{\mathrm{c}}$.

Successful photopolymerization relies on the effective initiation step, so that the photointiator (PI) is a critical component of a polymerizable formulation to reach the final cross-linked product. The vast majority of photoinitiating systems are based on free radicals as reactive species. Commercially available photoinitiating systems with different specific maximal absorption wavelengths $\left(\lambda_{\max }\right)$ and corresponding molar extinction coefficients $(\varepsilon)$ were studied [96,112]. The typical examples of PIs sensitive in UV range are 2,2diethoxyacetophenone (DEAP, $365 \mathrm{~nm}$ ) and 2,2-dimethoxy-2-phenylacetophenone (DMPA, $365 \mathrm{~nm}$ ). Sensitivity of the photoinitiating system can be extended to the visible range either by adding a suitable co-sensitizer (e.g. 4,4'-bis(dimethylamino)benzophenone, 300-500 nm) to one of the aforementioned PIs or by using the typical visible light photoinitiating systems such as camphorquinone, ethyl $4-\mathrm{N}, \mathrm{N}^{\prime}$-dimethylaminobenzoate, trethyl amine, isopropyl thioxanthone $(470 \mathrm{~nm})$. A considerable attention has been paid to the studies on the PIs sensitive in near infrared range $(>700 \mathrm{~nm})$. For example, cyanine dye-borate complexes have been studied [113] as promising PIs with $\lambda_{\max }=780 \mathrm{~nm}$. The near infrared sensitive PIs give a possibility to use cheap, compact and easy operational laser diodes and polymer fibers, which are less fragile and more robust and rugged than UV transparent ones.

Because of differences in chemistry for synthesis of hydrogels with the desired end properties, two or three different initiators are suggested to be tested. As an example, Figure 11 shows the photopolymerization kinetics for two hydrogel forming solutions with the same monomer (NIPA, $5 \mathrm{wt} \%$ ) and cross-linker (MBA, $0.5 \mathrm{wt} \%$ ) content, but different PIs DEAP and DMPA. The time course of light intensity at $\lambda=365 \mathrm{~nm}$ and $391 \mathrm{~nm}$ scattered at a $90^{\circ}$ angle from the polymerizable formulation shows that photoinitiating activity of DMPA is higher than that of DEAP under the same conditions. Indeed, polymerization started three times earlier when initiated by DMPA than DEAP (arrows).

The concentration of PI should be also optimized because of the following reasoning: on the one hand, the high concentration of PI provides a reliable polymerization with a high rate. On the other hand, too high rate and high level of cross-linking near the surface does not allow the light effectively penetrate farther inside the hydrogel forming solution. Moreover, limited water solubility of PI may lower its initiating efficiency and increase heterogeneity of the resultant polymer network. Solubility in water may be improved by dissolving the initiator in $70 \%$ ethanol solution before adding to the polymerizable formulation. The range of reasonable concentrations for DEAP was found to be from 0.01 to $1 \%$, whereas for DMPA it is 3 times lower due to a higher extinction coefficient. 


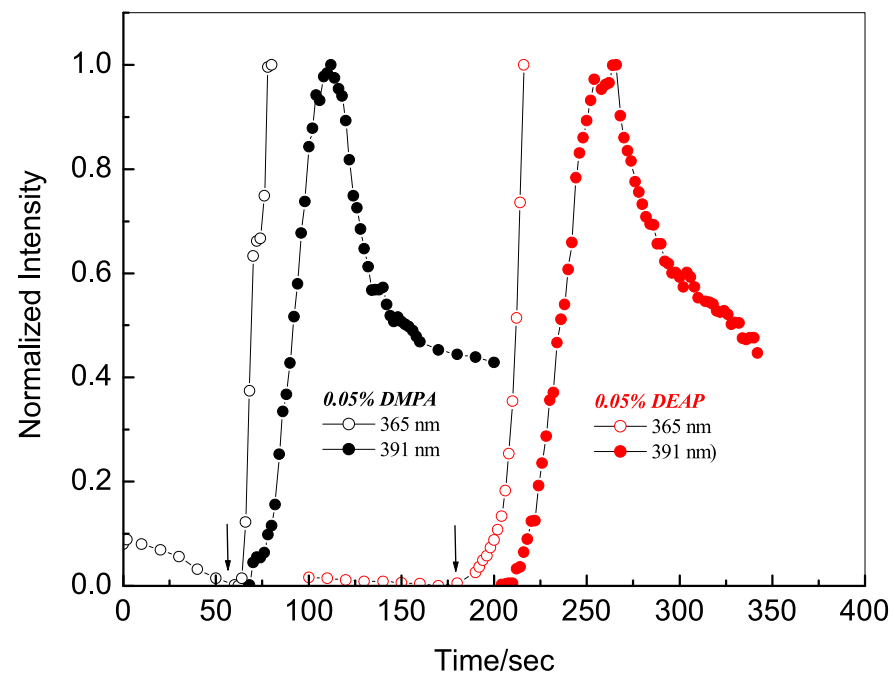

Figure 11. Polymerization kinetics of bulk PNIPA hydrogel with two different photoinitiators $-2,2^{\prime}-$ diethoxyacetophenone (DEAP, red circles) and 2,2'-dimethoxy-2-phenylacetophenone (DMPA, black circles) measured by intensity of two spectral lines $365 \mathrm{~nm}$ and $391 \mathrm{~nm}$ ) scattered perpendicular to the light initiating the polymerization. Diffuse radiation of Blak ${ }^{\circledR}$ Ray mercury lamp ( $\left.365 \mathrm{~nm}, 100 \mathrm{~W}\right)$ was used for the photopolymerization. Arrows show the instant when polymerization starts.

\subsection{Optical fiber preparation and reaction chamber design}

When selecting an optical fiber, it is important to work towards its transparency in a required spectral range, maximizing the light intensity collected into the fiber, and optimizing the length of the cladding-free area to provide a sufficient average path length within evanescence area for detectable level of an evanescent wave spectrum.

A plastic clad silica optical fiber with a high $\mathrm{OH}$ content is needed for $\mathrm{UV}$ and visible transmission. Since the light penetrates $\sim 0.1 \lambda$ into surrounding polymer, the depth of evanescent field penetration varies from $\sim 30$ to $70 \mathrm{~nm}$ for $300-700 \mathrm{~nm}$ wavelength range. The systematic studies show that the total path length for spectral measurements should be at least on the order of a micrometer. An appropriate path length necessary for an evanescent wave spectrum recording is determined by the portion of fiber with removed cladding. Herein, the critical angle of the total internal reflection and number of reflections depend on the core diameter $(\varnothing)$ and numerical aperture (NA) of a selected fiber: For example, for a fiber core with $\varnothing 600 \mu \mathrm{m}$ and NA $=0.48$, a 4-cm-long cladding free area provides a path length of $\sim 1.6 \mu \mathrm{m}$ for an evanescent wave with $\lambda=365 \mathrm{~nm}$.

Based on this estimations the reaction chamber has been designed (Figure 12). A 50-cm length SMA-SMA ending fiber cord (BFH48-600, Thorlabs Inc., Newton, NJ) having a 600$\mu \mathrm{m}$ core diameter and numerical aperture of 0.48 was used. This hard polymer clad multimode fiber with high $\mathrm{OH}$ content is transparent in the spectral range from 300 to 1200 
$\mathrm{nm}$. The evanescence area is prepared by removing a plastic clad in the middle of the cord. In general, there are three methods known for the removal of the cladding on different types of fibers: mechanical, thermal, or chemical. A chemical cladding-removal technique would be ideal, since it greatly reduces the possibility of mechanical damage to the surface of the fiber core. However, a 30- $\mu \mathrm{m}$ thickness polymer cladding of this fiber is too hard to be dissolved in any solvents in a reasonable time. Mechanical methods also are not reliable due to fragility of the fiber core. The fiber cladding was removed by a burning method using a propane or butane burning torch. When the fiber is stripped from the buffer, it should be held by an appropriate fixture in which fiber can rest and which protects that portion of the fiber core after cladding removal. The design of the reaction chamber (removable bottom cover) allows it to be used as the fiber fixture during the burning process and as a cleaning bath after. The surface of fiber core free of polymer cladding thoroughly cleaned with isopropyl alcohol, distilled water, and finally, compressed air to remove any carbonization. The quality of the surface was controlled under an optical microscope.

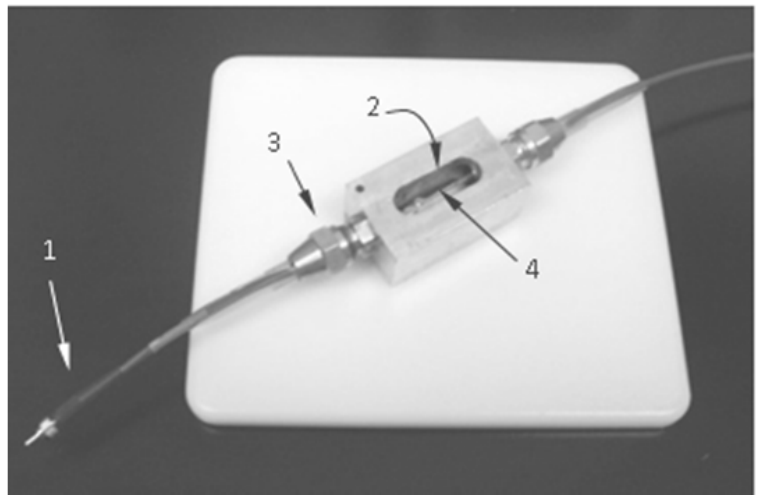

Figure 12. The reactor-container: (1) SMA ending fiber cord, (2) reservoir containing a polymerizable formulation ( 7 mL), (3) sealing gaskets, (4) fiber core without cladding exposed to a hydrogel-forming solution.

The recent studies suggested [114] that evanescent field can be pronounced and sensitivity of FEWS can be drastically increased by decreasing the diameter of fiber core. Since the extra caution should be taken with fibers under $200-\mu \mathrm{m}$ diameter, the evanescent wave cuvette of a new design was developed (Figure 13). Now, the so-called detection element is a movable module made of a cladding-free fiber core with the SMA compatible connectors. The distal ends of the detection element are polished to maximize the intensity of light passing through the fiber core. The modified reaction chamber offers the following advantages: maintains the integrity of the evanescence area, offers the feasibility of handing at all steps (polymerization, cleaning, detection, characterization), suits to a broad range of applications, such as a sensor with specifically modified functionality, and allows real-time watching the evanescence area during polymerization. 


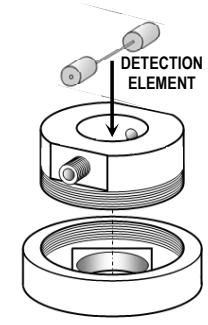

UPPER AND B OTTOM PARTS OF THE REACTION CHAMBER

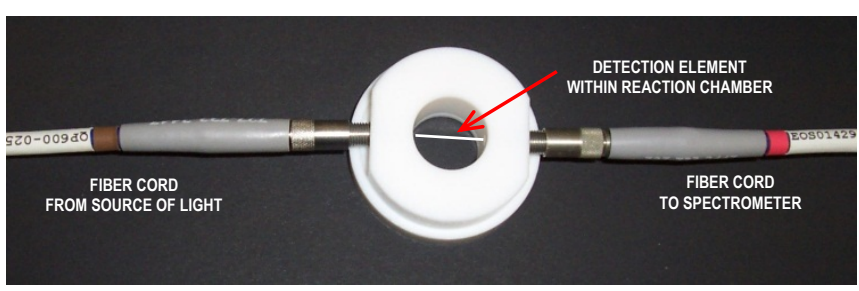

Figure 13. Schematic (a) and manufactured (b) the evanescent wave cuvette of a new design for polymerization on a side surface of the fiber core and recording the hydrogel swelling/de-swelling kinetics. The status of the hydrogel on fiber core can be controlled microscopically in the course of polymerization and swelling/de-swelling. Detection element is a removable part allowing for characterization by the other methods (e.g. atomic force microscopy).

\subsection{Instrumentation}

Light sources. Of equal importance to the PI, however, is the light source. Basically, two types of light sources are available: arc lamps, lasers, and light-emitting diodes. Recent advantages in light source technology are the development of lamps, lasers and/or diodes which offer increased light intensity at specific wavelengths. Regardless of the type of light source the emission spectra of the source must overlap the absorption spectrum of the chosen initiator. In this work, the Oriel Research Arc Source (Newport Corp., NJ) with a condenser, built-in ignitor, and $200 \mathrm{~W} \mathrm{Hg-Xe} \mathrm{or} 100 \mathrm{~W} \mathrm{Hg}$ lamp was used for the purposes of PEW on optical fiber. A Blak Ray mercury lamp (365 nm, $100 \mathrm{~W}$ ) was used for polymerization of a bulk hydrogels. A Deuterium-Tungsten halogen light source (DT-MINI2) from Ocean Optics was used for spectroscopic detection and characterization of hydrogel films on the fiber core.

Fiber-optic spectrophotometers. Fiber-Optic CCD Array UV-Vis Spectrophotometer (Ocean Optics, Inc.) with a convenient fiber optics connectorization, high sensitivity, high recording speed (1 spectrum/sec), broad wavelength range (180-900 nm), and high spectral resolution $(<1 \mathrm{~nm})$ was used for routine spectroscopic measurements. SpectraSuite software was used to record, treat, and store radiation, transmission and absorption spectra.

Optical microscopy. Digital Optical Microscope (DC3-163-PH, Microscope World, CA) with Imaging Software (Motic Images 2000, Motic Group, Ltd.) was used to measure the diameter of the bare fiber core and thickness of the cylindrical hydrogel on the fiber core after washing. To detect and characterize a relatively thick hydrogel film $(>10 \mu \mathrm{m})$ on fiber core after polymerization or during swelling/de-swelling, an optical microscope with low magnification was used as well. 


\subsection{Experimental setup for photopolymerization on side-surface of optical fiber core}

The effect of radiation intensity and exposition time on the hydrogel film formation by evanescent wave photopolymerization was examined using the $200 \mathrm{~W} \mathrm{Hg-Xe} \mathrm{lamp.} \mathrm{The} \mathrm{UV}$ light was focused with a condenser and collimating lenses on SMA905 ending fiber, which directs the light through the bandpass filter to the cladding-free portion of fiber core fixed within the chamber containing a polymerizable solution as shown in Figure 14. The other SMA end of the fiber cord was connected through an attenuator to a fiber optic spectrophotometer (USB4000-UV-VIS) with CCD array, which allowed for observing the spectrum of light transmitted through the whole pathway. The spectra recorded in the range from 180 to $900 \mathrm{~nm}$ were stored in a $1 \mathrm{sec}$ time interval.

The reaction chamber was placed into a temperature insulating box to provide a temperature control $\left( \pm 0.1^{\circ} \mathrm{C}\right)$, A temperature sensor was dipped into the reactor-container nearby the fiber core.

When the hydrogel forming solution was poured into the reaction chamber in the amount necessary to cover the fiber core, light was on. Passing through the fiber core, it causes evanescent waves penetrating outside to initiate polymerization in the close vicinity to the fiber core. Any changes in the intensity of light transmitted through the fiber are recorded by the fiber optic spectrophotometer which serves as a light detector. The PNIPA hydrogel film synthesized on fiber core was washed in deionized water for 3 days at room temperature before further characterization. It was found that thickness of the hydrogel film could vary from nanometer scale $(<1 \mu \mathrm{m}$, cannot be detected by optical microscopy) to hundreds of microns by varying the dose of energy - the product of power of the light source and polymerization time (see section 2.2.3).

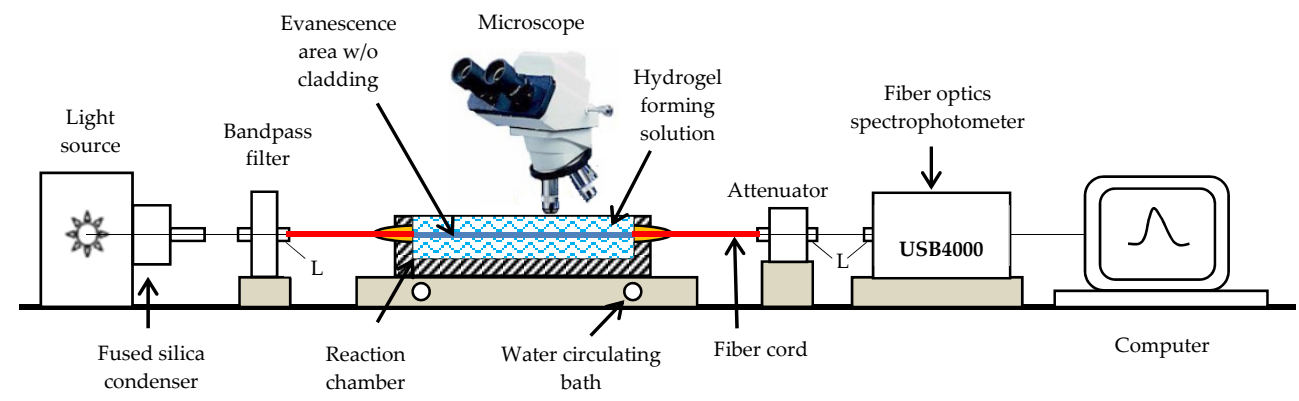

Figure 14. Optical scheme of the system for polymerization of cylindrical hydrogel on a side surface of the fiber core and recording the hydrogel swelling/de-swelling kinetics. L - lenses.

The same optical scheme (Figure 14) was used to examine the kinetics of hydrogel swelling/de-swelling in the course of film hydration and during the volume changes. The only difference was that a Deuterium-Tungsten Halogen light source (DT-MINI-2) provided 
continuous spectrum in the whole UV-VIS range from 180 to $900 \mathrm{~nm}$. The time course of integral intensity and spectral composition of light passing through the fiber core with hydrogel film on it was measured at different temperatures. Temperature control inside the reaction container was carried out permanently in the course of swelling kinetics study. Transmission or absorption spectra were saved each second when temperature stabilized on a certain level within the experimental uncertainty of $\pm 0.1^{\circ} \mathrm{C}$.

\section{Properties of hydrogel film on the fiber core}

\subsection{Hydrogel films of nanometer and micrometer thicknesses}

\subsubsection{Kinetics of photopolymerization of a thin hydrogel film on the fiber core}

To polymerize a thin PNIPA hydrogel on a side surface of the optical fiber core, a mercury $100 \mathrm{~W}$ lamp without condenser was employed as a light source. The lamp was turned on after a 15 min equilibration of the hydrogel forming solution within the reaction chamber at room temperature. The polymerization reaction was followed in situ by monitoring the integral intensity of light passing through the fiber core and recording it every 0.2 sec (sample time) using the above described setup (Figure 14). A typical integral intensity versus exposure time kinetic curve is shown in Figure 15.

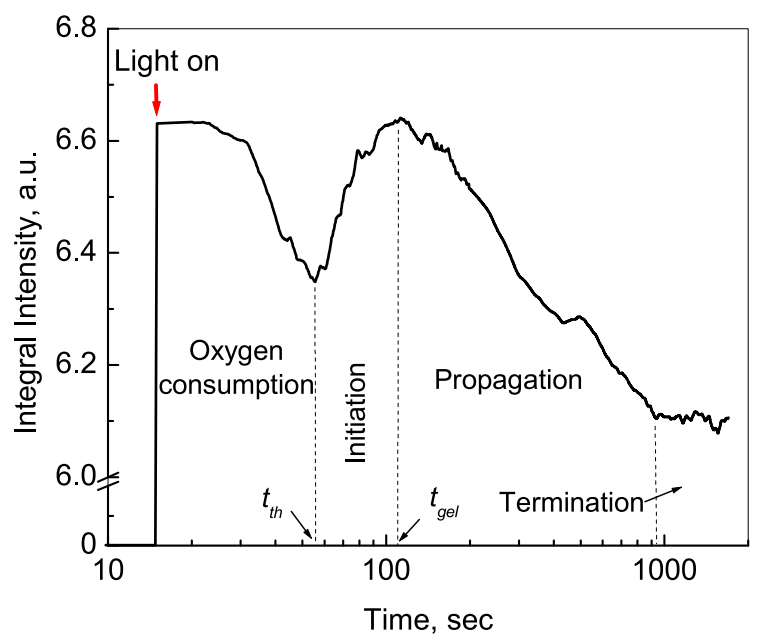

Figure 15. Time course of integral intensity during photopolymerization by evanescent waves on the fiber core surface: (UV lamp power $=100 \mathrm{~W}$, composition of the hydrogel forming solution is $5 \mathrm{wt} \%$ NIPA, 0.5 wt \% MBA, and 0.5 wt \% DEAP).

As it has been explained in the section 2.2.1 on the photopolymerization process, there is a delay between the beginning of irradiation and the formation of gel because the absorbed dose of energy is used not only for the decomposition of the initiator molecules into radicals, but also for the consumption of oxygen which deactivates the formed radicals. The pronounced decrease in integral intensity at the very beginning of UV illumination (red 
arrow) can be assigned to the oxygen consumption. When the concentration of oxygen decreases enough, the reaction of radicals with monomers competes successfully with the deactivation processes and the intensity of light returns to a higher level. Therefore, the minimum in integral intensity located at about 60 seconds $\left(t_{t h}\right)$ corresponds to the threshold dose of energy necessary to consume a sufficient amount of oxygen. The radicals react with functional groups of monomer and cross-linker forming a polymer, but absorbed dose of energy is not sufficient yet to reach the gel point. This happens only after $\sim 100$ seconds $\left(t_{g e l}\right)$, when a significant portion of evanescent light scatters on the polymer network as it becomes denser to decrease the integral intensity - propagation of polymerization starts. A further decay of the integral intensity continues until the thickness of hydrogel reaches the depth of penetration. At this step ( $\sim 15 \mathrm{~min})$, the average level of integral intensity becomes constant indicating that radicals react with each other and polymerization is terminated. The total polymerization time was $\sim 30 \mathrm{~min}$. Thus, the three key steps of polymerization kinetics (initiation, propagation, termination) have been distinguished.

\subsubsection{Hydrogel film of micrometer thickness on fiber core}

Theoretically, the thickness a hydrogel film on the fiber core polymerized by evanescent waves can be varied within the depth of evanescent field penetration out of the fiber core surface (typically $\sim \lambda / 10$ ). To examine experimentally how thick the hydrogel film can be formed, the intensity of UV light collected into the fiber and exposition time were maximized. The power of the UV light was increased by using the Hg-Xe $200 \mathrm{~W}$ lamp and fused silica condenser along with focusing lenses (Figure 14).

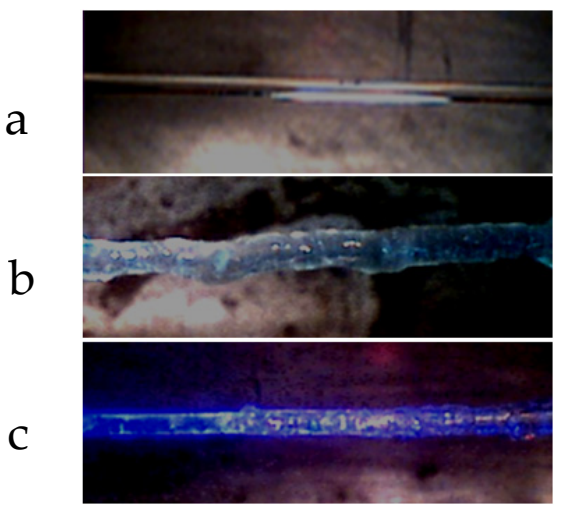

Figure 16. Optical micrographs (x10) of (a) bare fiber core on air, (b) hydrogel film on the fiber core on air after polymerization and washing, and (c) the same hydrogel film as for (b) dried overnight.

As in the previous experiment, before the UV light was allowed to pass through the fiber, thoroughly cleaned fiber core (Figure 16a) was completely covered with the hydrogel forming solution. The appearance of hydrogel was controlled by optical microscope and by measuring intensity of light passing though the fiber (polymerization kinetics) in real time. Figure 16b shows the cylindrical hydrogel film around the fiber core as a result of two hours of its exposition to UV light. It was a surprise that the average thickness of the film in wet 
state was $\sim 175 \mu \mathrm{m}$, i.e. remarkably thicker than the depth of evanescent wave penetration. After overnight drying on air, the thickness decreased up to $\sim 30 \mu \mathrm{m}$, still remaining thicker than the penetration depth (Figure 16c). In both cases, a blue glitter around the hydrogel cylinder is an indication that evanescent waves scatter within the hydrogel film. Herein, the intensity of light scattered inside the film in a wet state is much weaker than in the same film but dried overnight. Thus, the polymerization of hydrogel film of a macroscopic thickness, definitely, is the result of a successive scattering of evanescent waves on the newly formed polymer layers farther and farther from the surface of fiber core.

\subsection{Properties of environmentally sensitive hydrogel film on the fiber core}

\subsubsection{Detecting the hydrogel film of nanometer thickness by dry-wet swelling}

After measuring the polymerization kinetics as described in Section 5.1.1, the area exposed to polymerization was thoroughly and carefully washed in the reaction chamber by flowing distilled water at least for 48 hours. As expected, inspection of the fiber core surface under microscope did not allow us to prove the formation of a hydrogel film, since its thickness and the surface roughness was beyond the optical resolution. To detect the hydrogel film, the washed area of the fiber core was dehydrated by drying for 2 days on air. Then the measurements of the integral intensity of light passed through the fiber were carried out on air and after the addition of distilled water (see Sections 3.2 and 4.4 for details). Figure 17 shows three steps of changes: (i) an abrupt drop in intensity corresponds to the change in the refractivity of the fiber core/external medium interface, (ii) a very fast increase in the integral intensity due to immediate swelling of hydrogel in water, and (iii) a slow relaxation of the polymer network to equilibrium by smoothing up its microheterogeneities. Control experiments with bare fiber core did not show the later two steps. Together with polymerization kinetics data, these results unequivocally confirm the formation of a thin hydrogel film on the surface of fiber core.

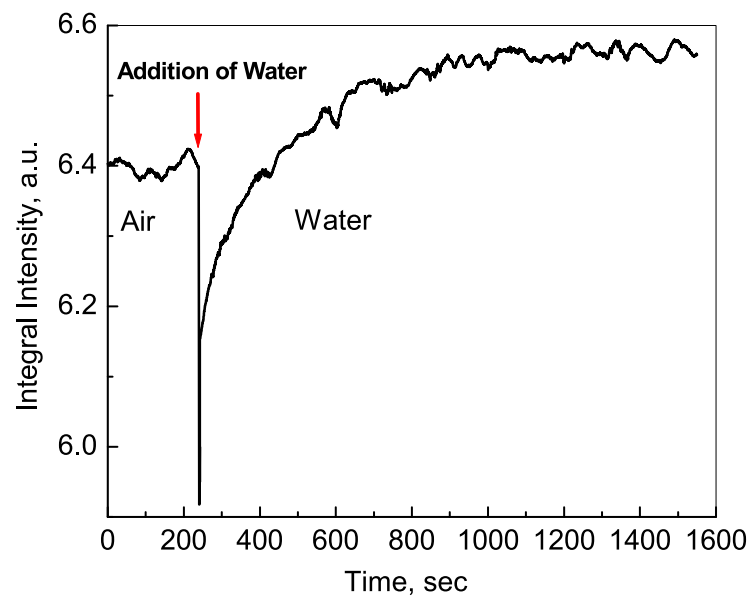

Figure 17. Time-dependence of the integral intensity of light passing through the fiber core during hydrogel film hydration on its surface. 


\subsubsection{Dry-wet transition in hydrogel film of micrometer thickness}

The macroscopic PNIPA hydrogel film (Figure 16) was washed in deionized water for 3 days and dried on air for 120 hours at room temperature. The kinetics of hydrogel swelling in the course of film hydration is shown in Figure 18. The process is a multistep one. As for the thin film, the three steps can be clearly seen: (1) penetration of water inside the gel to reach the fiber core surface, (2) swelling of the hydrogel throughout the entire thickness, and (3) a slow relaxation of the polymer network to the equilibrium state. Totally, fiber transmittance increases 2-fold as a result of the hydrogel swelling (hydration).

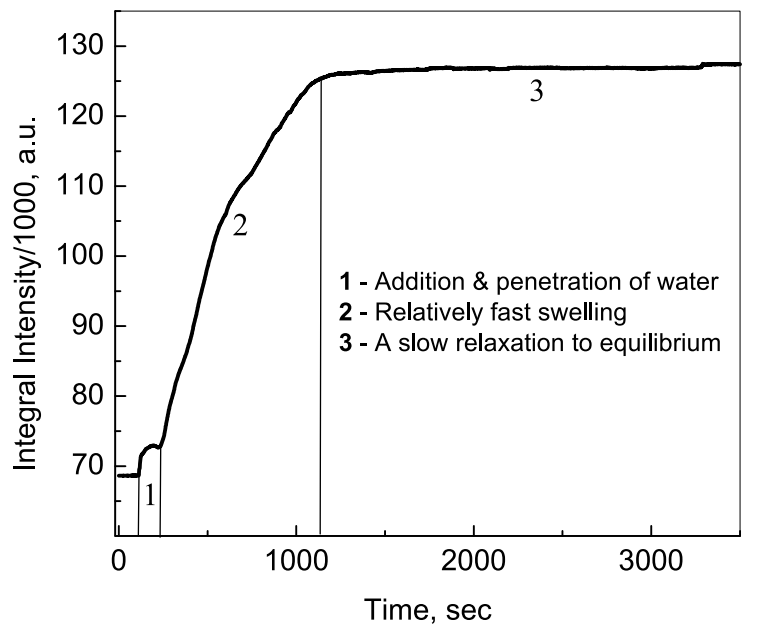

Figure 18. Integral intensity of light passed through the fiber core covered with dried micrometer PNIPA hydrogel film in the time course of its hydration.

\subsubsection{Temperature sensitive volume transition of hydrogel micrometer film on the fiber} core

The PNIPA hydrogel film on fiber core (Figure 16) was equilibrated at room temperature in the reaction chamber filled with distilled water. The hydrogel in water is in a swollen state. The integral absorbance $(200-880 \mathrm{~nm})$ in the fiber core was measured at different temperatures using the same scheme as described in previous section. Figure 19 clearly shows that absorption/scattering of evanescent light inside the PNIPA hydrogel film begins to increase at $\sim 32{ }^{\circ} \mathrm{C}$. This value is in good agreement with the transition temperature $\left(\mathrm{T}_{\mathrm{c}}\right)$ known for PNIPA hydrogels from the literature [110] and our previous data [103] as the socalled Lower Critical Solution Temperature (LCST) at which a temperature sensitive hydrogel in aqueous medium collapses. As a result, the portion of evanescent wave scattered on the dense polymer network increases. Assuming the linear proportionality between absorbance and density, the temperature sensitive PNIPA hydrogel film underwent more than 4 -fold volume decrease when temperature increased from 32 to $55^{\circ} \mathrm{C}$. Absorbance in the bare fiber core without any hydrogel has been measured for comparison, to illustrate that changes are attributed to the hydrogel layer collapsing. 


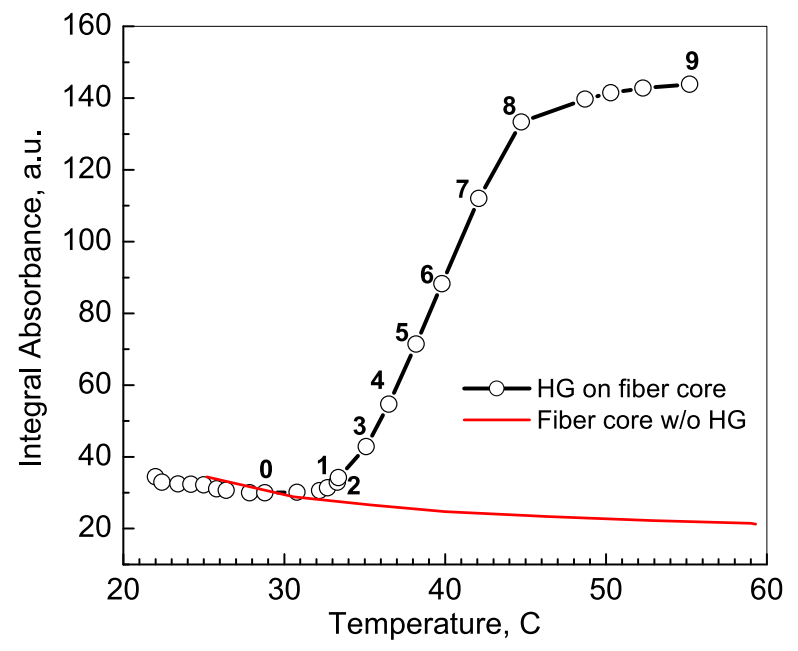

Figure 19. Integral absorbance $(200-880 \mathrm{~nm})$ in fiber core with and without PNIPA hydrogel film as a function of temperature.

Importantly, the continuous (without abrupt steps) changes in fiber transparency in the course of both dry-wet and temperature sensitive volume transitions indicated that the gel polymerized on the fiber core was tightly adhered to the surface without losing a contact at swelling/contraction. Applications of hydrogel spots on fiber distal ends and on a fiber core as chemical and biochemical sensors also confirm an excellent adhesion of hydrogel to the fiber surface.

\subsubsection{New spectral properties of the temperature sensitive hydrogel film on the fiber core}

The hydrogel/fiber core assembly proposes the experimental scheme which allows not only the integral intensity, but also the transmission (or absorption) spectra to be recorded in the whole range of wavelengths. For the first time, the spectral composition of light passing through the fiber with PNIPA hydrogel on it has been analyzed at different temperature in the course of the polymer network collapsing. The absorption spectrum at $28.8^{\circ} \mathrm{C}$, below the volume transition temperature, was taken as a reference (0) with respect to which the spectra at higher temperatures are represented in Figure 20.

Strikingly, the spectral range of absorption depends on temperature, widening with the extent of temperature increase above $T_{c}$. Herein, maximum of absorbance shifts more and more to the longer wavelengths with temperature. Finally, at temperatures higher than $55^{\circ} \mathrm{C}$, absorption was maximized in the whole range from 200 to $900 \mathrm{~nm}$ (in red) enveloping the absorption ranges at lower temperatures. This process was reversible, reflecting the reversibility of the volume transition.

The observed variations in the absorption range with temperature can be explained by scattering of evanescent waves [115] on growing microheterogeneities of the polymeric network when it collapses. At the volume transition temperature $T_{c}$, the size of 
heterogeneities is small, so that only light with short wavelengths considerably scatters, contributing to the absorption range of the recorded spectrum. In the course of temperature rising, the heterogeneities grow and new spectral components of evanescent light with longer wavelengths begin to scatter - the spectral range of absorbed light expands from ultraviolet to visible and further to the near infrared domain. Moreover, one can expect that concentration of growing heterogeneities also increases with density of collapsing polymer network. At some instant, the scattering features of evanescent light changes from Rayleigh to Mie scattering $[15,116]$.

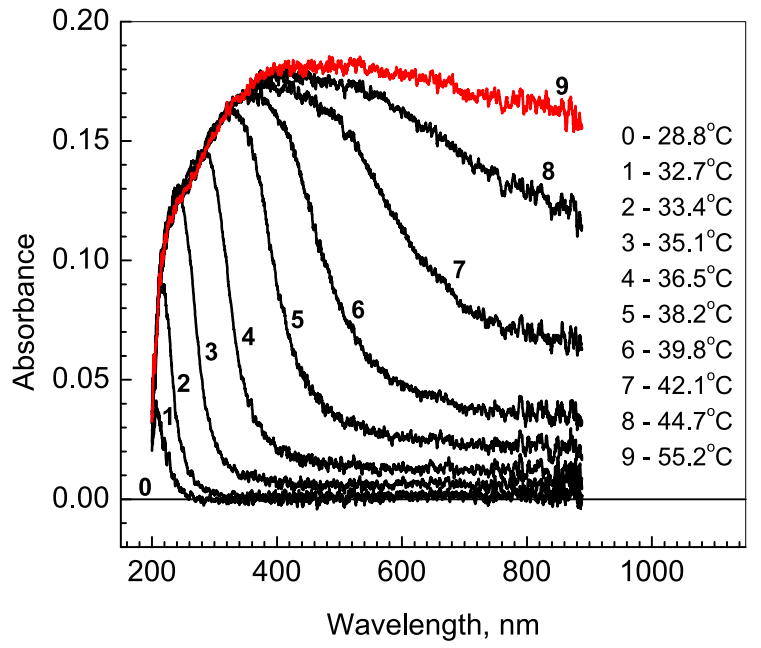

Figure 20. Absorption spectra of the fiber core covered with the cylindrical PNIPA hydrogel (PNIPA) at different temperatures indicated and numbered in accord with points labeled in Figure 19.

\section{Future applications of hydrogel film/fiber core structures}

\subsection{Scientific and practical aspects of photopolymerization, detection, and characterization}

The main reason of focusing on optical fiber core is that being a source of evanescent waves it provides the spectroscopic access to the surrounding medium. Connected to a spectrometer, an optical fiber with open core becomes an apparatus for polymerization and analytical device in one.

Since polymer networks of different chemical origin are increasingly studied for applications in biosensing, drug delivery and tissue engineering, their polymerization kinetics will be examined using the evanescent wave spectroscopy on the fiber core. The diversity of polymerizable formulations will initiate the development of new photoinitiating systems for reliable polymerization by visible and near infrared light.

Controllable formation of hydrogel nanoparts of different shapes and sizes on side surface of a fiber core is another direction of the future studies. Good quality and reproducibility of 
the nanoparts patterning on cylindrical support is a specific aim of the research on PEW. The development of technology for optical fibers with sub-wavelength diameter will drastically increase the evanescent field power and resolution of cylindrical nanopatterning.

Once hydrogel film is formed on a fiber core, its swelling/shrinking kinetics should be compared to theoretical predictions. However, quantitative kinetic model of swelling/deswelling is still in demand, especially on a submicrometer scale. Despite some of the kinetic approaches to hydrogel swelling $[117,118]$ exist, the changes in spectral composition of evanescent light scattered within polymer networks affected by external stimuli, chemical composition, dimensions, and load have never been able to measure.

Immobilization of chemical and biological specificity on the hydrogel cylindrical nanofilms is the other aspect of the future studies. A possible scheme for introduction of a polypeptide specific to biological moieties into polymer matrix of nanofilm developed in conjunction with bacterial spore interaction with liposomes [119] is shown in Figure 21. At Step 1, amine-groups $\left(\mathrm{NH}_{2}-\right)$ are incorporated into hydrogel film by co-polymerization of $\mathrm{N}$-isopropylacrylamide with $\mathrm{N}$-(3-aminopropyl)methacrylamide in the presence of $N, N^{\prime}$ methylenebisacrylamide (cross-linker) and 2,2-diethoxyacetophenone (photoinitiator). Polypeptide-hydrogel conjugation is performed with heterobifunctional cross-linker [85,86], N-succinimidyl 3-(2-pyridyldithio)propionate (SPDP), as shown in Figure 21 (Steps 2 and 3).

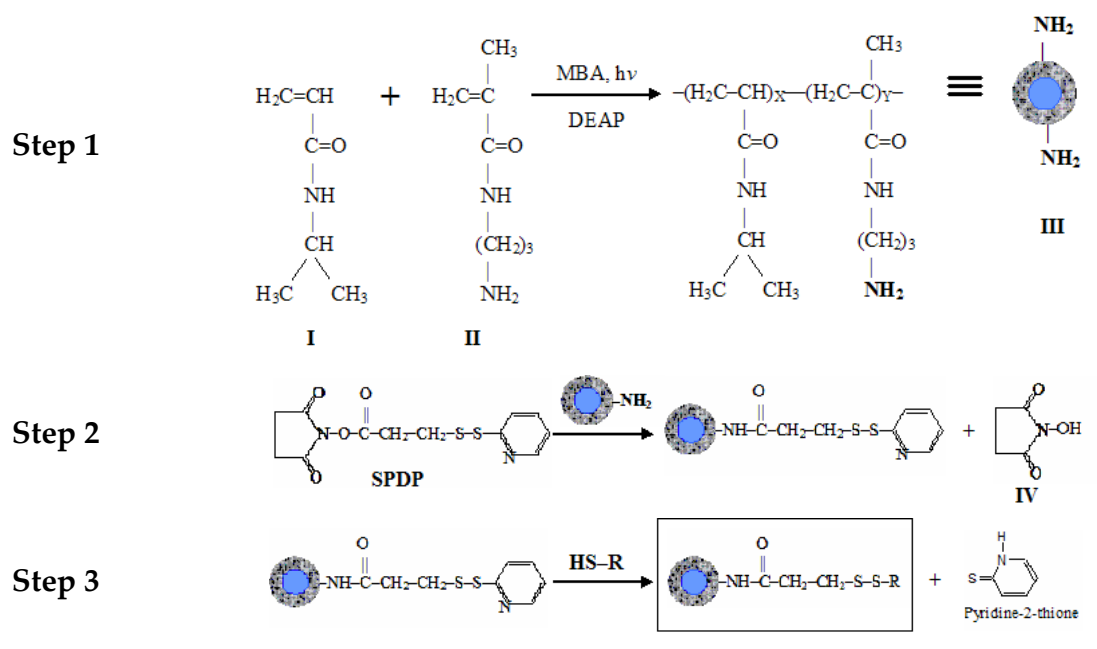

Figure 21. Polypeptide-hydrogel conjugation: (Step 1) Photo-co-polymerization of $\mathrm{N}$-isopropylacrylamide (I) and $\mathrm{N}$-(3-aminopropyl)methacryl-amide (II) on the surface of a fiber core. The amine-groups are everywhere in the bulk of hydrogel including the surface of the nanofilm. (Step 2) Preparation of pyridyl disulfide-activated surfaces by aminolysis leading to the release of $N$-hydroxysuccinimide (IV) and formation of an amide bond. (Step 3) Polypeptide (R) coupling to hydrogel nanofilm. 


\subsection{New materials: Quasi-two-dimensional network}

The proposed cylindrical architecture of hydrogel film on side surface of fiber core may revolutionize conventional applications of supported polymer matrices. The use of evanescent waves for preparation of a cylindrical ultrathin hydrogel film on the side surface of a fiber core is a promising method for thickness control on nanometer scale. The dosage of energy (intensity times polymerization time) can be optimized in an attempt to get a new class of materials - quasi-2D-polymer webs (Figure 22).

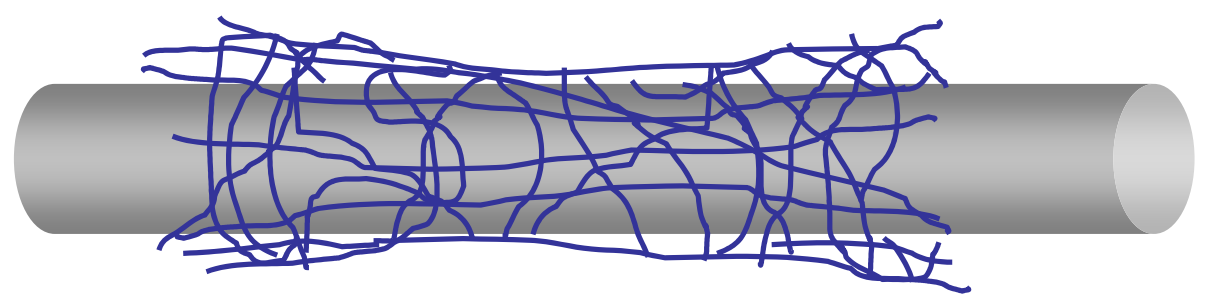

Figure 22. Cylindrical quasi-two-dimensional polymer web on optical fiber.

Future applications of the "ultrathin film/fiber core structures" originate from the unique properties of the ultrathin hydrogel film, namely: (1) the world's highest level of expansion and contraction, (2) the most sensitive externally induced mass transfer, and (3) the record fastest response to different stimuli. Therefore, the cross-linked polymer matrix of nanometer thickness on a fiber core is of great potential for a much broader spectrum of applications than just sensors. Generally speaking, the intersection of optical fiber technology and polymer nanotechnology offers numerous new science and technology opportunities. For example, the cylindrical sheet (Figure 22) prepared from a conductive polymer is expected to be stable, highly flexible, strong and remarkably conductive, so that electrons can travel with small scattering over submicron distances. Those cylindrical nanofabrics are important for making very-fast-switching transistors leading to biological computers which can use living cells and microorganisms as high memory density elements [120].

\subsection{Modeling cell-like structures}

Recent successful approaches to the development of new models of biological membranes by designing solid-supported phospholipid bilayers have demonstrated the importance of a water-filled space between the solid substrate and bilayer [121-125]. Up to now, only the planar architecture of the model has been considered. The proposed nanofilm/fiber structure might be an initial step in building a cylindrical model of biological membrane which is named "shish kebob structure" (Figure 23). We believe that the cylindrical architecture is a starting bottom-up polymer-chemistry-driven model of intracellular organization with the possibility to monitor biochemical processes spectroscopically within the cell, thereby revolutionizing cell biology. 


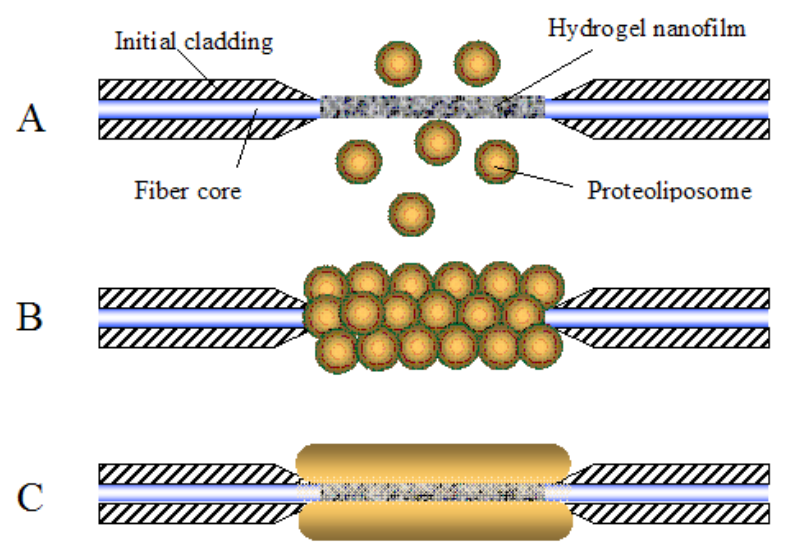

Figure 23. "Shish kebob structure" as a cylindrical polymer-driven model of a biological membrane: A) the preliminary prepared nanofilm/fiber structure is exposed to the proteoliposome suspension, B) the proteoliposomes adhere on the appropriately modified surface of the hydrogel ultrathin layer, C) spontaneous fusion of the proteoliposomes and formation of the cylindrical bilayer supported by the nanofilm/fiber structure.

\subsection{New types of biosensors: From chemicals and biological agents to biofilms and single cell}

The proposed cylindrical hydrogel/fiber core structure is a bicompartmental (optical and chemical) spectroscopic tool which can be specifically designed for accurate monitoring/detection of chemicals and living entities within "microscopic" environment, particularly, within a single cell or microorganism.

The optical part (fiber core) allows the monitoring of biochemical processes if not within the single cell, then within a biological community, and manipulating of cellular processes by transmission of information into biological systems, for example, using different wavelengths of electromagnetic radiation delivered by the fiber into the cell as shown in Figure 24 .

The chemical part provides the following features: (i) hydrogel is a suitable semiwet 3Denvironment for biological interactions, (ii) many hydrogels are inert surfaces without nonspecific adsorption of proteins (antifouling), (iii) biological molecules can be covalently incorporated into hydrogel using different chemistries (e.g. see Figure 21), (iv) mechanical properties of hydrogel materials are tunable by modifying cross-linking density, and (v) hydrogel can be responsive to external or internal stimuli (see Introduction).

Imagine Figure 23B where proteoliposomes are substituted for microorganisms. The proposed structure looks extremely potential for application as a microbial biofilm microorganisms' culture growing on a supported polymeric matrix [126]. Cells within a biofilm are embedded in a matrix of the so-called extracellular polymeric substances (EPS), primarily produced by the microorganisms themselves. Little is known about this matrix. 
The composition of EPS varies depending on the organisms present and environmental conditions. Characterization of EPS matrix is a daunting proposition.

The ability of bacterial spores to coordinate their alert sensory behavior and processes to respond to the germination effectors [119] are increasingly studied. However, several key features of sporulation and germination in bacteria are not understood at the molecular level. Presumably [127-129], under proper pre-germination circumstances, the spores can and will attempt to influence their surrounding chemical and physical environment by secreting specific biological macromolecules and solutes. The challenge is in determining when, why and how the bacterial spore population regulates external matrix composition and what the ultimate functional consequences are. It would be especially interesting to deploy spores on specifically modified hydrogel/fiber core structure.

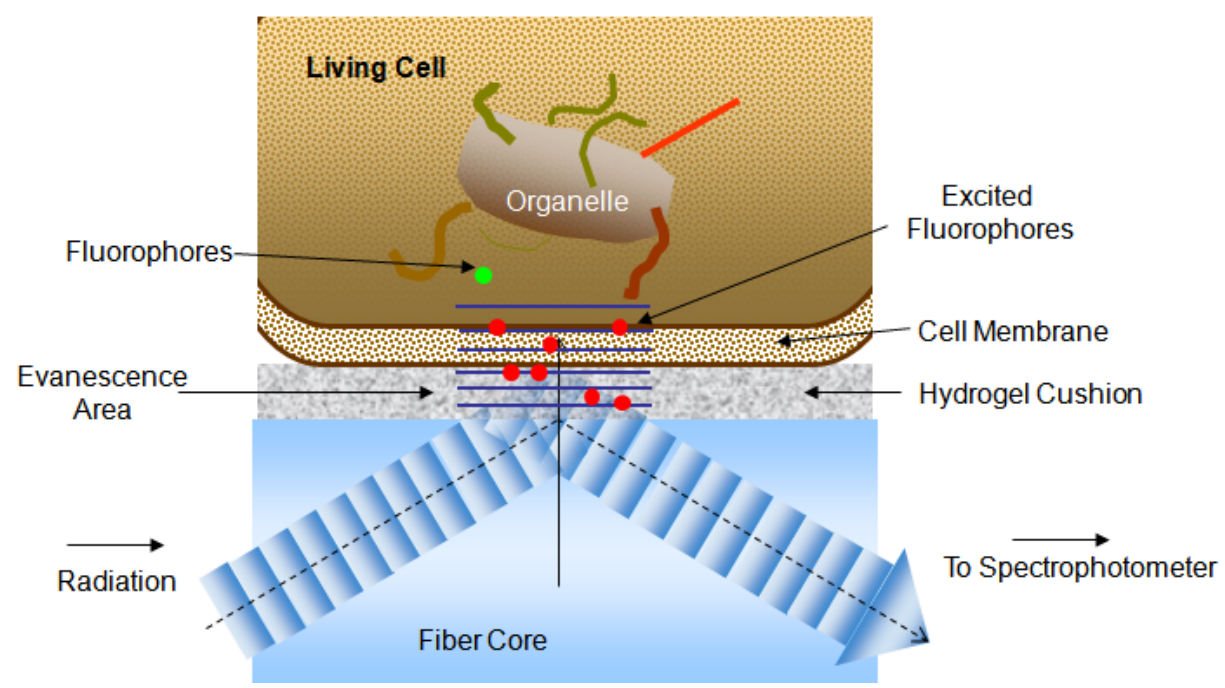

Figure 24. Single cell supported by cylindrical hydrogel film on optical fiber core.

Evanescent field penetrates through the hydrogel cushion and membrane into the living cell either for detection or initiation of biochemical processes inside. With fluorescent probe or without, spectroscopic signal can be recorded.

Both for dormant (spores) or living cells, fiber core can serve as an optical guide for recording spectroscopic information on the structural transformations and chemical changes in real time, whereas responsive hydrogel material can be modified to provide triple functionality: (i) the cell-adhesive ligands (e.g. polypeptides [119]) can be incorporated into the so-called bioselective hydrogel to adsorb a certain type of cells (spores), (ii) the bioresponsive hydrogels can be designed to contain bioactive ligands (peptides, polysaccharides) to direct cell behavior (germination, secretion, differentiation, division, etc.) [130-133], and (iii) the biointeractive hydrogel can be modified to contain receptors (proteins, antibodies, enzyme-sensitive substrates, enzymes) for biomolecules or cells that, when stimulated, trigger local or bulk changes in the hydrogel properties (swelling/collapse) [134-137]. 


\subsection{Tissue engineering}

Hydrogels have been widely used in tissue engineering [130-138]. Due to their biocompatibility, ability to simulate the properties of living tissue, and controlled diffusivity of nutrients and methabolites, hydrogels are versatile scaffolds which can support the implanted cells. The whole pool of recent study of 3D hydrogels scaffolds for tissue engineering puts forth a hypothesis that the thinner is the hydrogel scaffold, the more effective are the processes of cell differentiation and growth, presumably, because of the enhanced mass transfer in thin films. Thus, nanofilm/fiber structures may offer significant improvement over the current methods of tissue engineering providing the most sensitive biological, chemical, and physicomechanical response of ultrathin hydrogel film. Furthermore, nanofilm/fiber structures offer the capability of spectroscopic controlling the properties of a functional tissue in the course of cell growth on the side surface of an optical fiber.

\subsection{Drug delivery devices}

The nanofilm/fiber structure with drug filled polymer matrix on the side surface of the fiber core can be inserted into the organ of interest. The battery of such kind of structures can be patched on the skin. Both configurations of the drug delivery devices can provide methods for triggering the drug release and for spectroscopic control of the delivery dose. Numerous techniques [2, pp. 95-126] have been used for immobilizing drugs on/within a hydrogel: physical entrapment, electrostatic attraction, physical adsorption, and/or chemical bonding. The polymerization of hydrogel by evanescent waves in the presence of a drug offers a new method for entrapping the drug into the ultrathin hydrogel film. Thus prepared nanofilm/fiber structures could be a key step in designing a combined drug delivery systems with precise quantification of a delivery dose: (1) various drugs entrapped in different structures can be simultaneously or in desired order released in the targeted organs in the body; (2) several nanofilm/fiber structures can be loaded with different predrug reagents (non-harmful for surrounding organs) which are allowed to react once being delivered to the targeted site, thereby the surrounding organs would be protected against the final form of the drug.

\section{Closing remarks and conclusions}

The increasing amount of studies has already demonstrated that environmentally responsive hydrogel materials could be successfully used for detection of chemicals, biological compounds, living cell, and microorganisms, for targeted delivery of drugs in response to disease-specific biomolecules, and for triggering cell behavior and tissue repairing. Traditionally, only the planar design of these "smart" polymer networks has been considered. In this paper, cylindrical configuration of responsive hydrogels supported on side-surface (not distal end) of an optical fiber core was introduced. It was shown that absorption/scattering of the evanescent field penetrating out of the fiber core surface (typically, $\sim \lambda / 10$ ) can be feasibly used not only for the fabrication of the responsive hydrogel film around the cylindrical surface of fiber core, but also for recording the response of thus 
deposited soft material to environmental changes. Photolymerization by evanescent waves is a versatile and convenient method for synthesizing hydrogels films with thicknesses varied from nanometers to hundred micrometers. A simplistic model of PEW predicts that within the depth of evanescent field penetration out of the fiber the thickness is controlled by the dosage of absorbed energy (light intensity $\times$ polymerization time). The thicker hydrogel films are due to scattering of evanescent waves on the newly formed polymer microstructures and initiation of polymerization relatively far from the surface of fiber core.

The designed bicompartmental spectroscopic tool of cylindrical configuration allows one to use the same experimental scheme to polymerize, to study the kinetics of polymerization, to detect and characterize the deposited polymer network simultaneously. One major new option that was not previously available is the capability of real time analyzing the spectral composition of light absorbed/scattered within a hydrogel during polymerization, swelling/de-swelling, and other macroscopic changes in response to external stimuli or triggered internal interactions.

The option of spectral analysis was demonstrated on swelling kinetics of the temperature sensitive polymer network of poly( $N$-isopropylacylamide) synthesized around fiber core in the course of film hydration and the gel temperature sensitive volume transition. Particularly, the hydrogel supported by fiber core began to absorb/scatter light in ultraviolet region at the temperature of volume transition $\mathrm{T}_{\mathrm{c}}$. However, the spectral range of absorption continued to expand into the visible and farther to the near infrared domains while temperature was getting higher.

Polymer network formed is not covalently bound to fiber side-surface. It is a kind of "cylindrical sock" on the fiber core. Its structure and responsiveness depend on polymer chemistry whereas its thickness is a function of polymerization kinetics. Since the sensitivity of the method is expected to increase with fiber core of sub-micrometer diameter, the hydrogel/fiber core nanostructures will be especially useful for testing of nanometer-sized biological objects and spectroscopic study of an individual living cell. We believe that further research on optimization of the non-planar (cylindrical) design of supported bioresponsive and biointeractive hydrogel nanoparts will bring us to a stage where we better understand both potentials and limitations of the approach in order to compete with that induced by conventional flat nanopatterning in the market.

The approach is generic - any soft biomaterials (pigments, surfactants, liposomes, lipobeads, proteins, polysaccharides, biomembranes, blood, cells and their organelles, spores, and even organisms) could be supported on the side surface of an optical fiber core and the study of their structural changes using the evanescent wave spectroscopy would benefit from the development of the hydrogel/fiber core nanostructures.

This is not a complete review on possible applications. Nevertheless, the aforementioned examples allow one to conclude that the methods for hydrogel nanofilm preparation and support on a fiber core of sub-micrometer diameter are in demand. 


\section{Author details}

Sergey V. Kazakov

Department of Chemistry \& Physical Sciences, Pace University, Pleasantville, NY, USA

\section{Acknowledgement}

This work was financially supported in part by Pace University (Dyson College of Arts and Sciences, Summer and Scholarly Research Funds). Acknowledgement is made to the Donors of the American Chemical Society/Petroleum Research Fund for partial support of this research (PRF grant \# 44161-GB5).

\section{References}

[1] Harland RS, Prud'homme RK (Eds.) (1992) Polyelectrolyte Gels: Properties, Preparation, and Applications. Washington, DC: American Chemical Society.

[2] Peppas NA (1987) Hydrogels in Medicine and Pharmacy. Boca Raton, FL: CRC Press.

[3] Galaev IY, Mattiasson B. (1999) Smart polymers and what they could do in biotechnology and medicine. Trends Biotechnol. 17: 335-340.

[4] Hennink WE, van Nostrum CF (2002) Novel cross-linking methods to design hydrogels. Advanced Drug Delivery Reviews 54: 13-36.

[5] Erman B, Flory PJ (1986) Critical phenomena and transitions in swollen polymer networks and in linear macromolecules. Macromolecules 19: 234-252.

[6] Pelton R (2000) Temperature-sensitive aqueous microgels. Adv. Coll. Interface Sci. 85: 133 and references therein.

[7] Tanaka T, Fillmore D, Sun ST, Nishio I, Suislow G, and Shah A (1980) Phase transitions in ionic gels. Phys. Rev. Lett. 45: 1636-1639.

[8] Eichenbaum GM, Kiser PF, Simon SA, Needham D (1998) pH and ion-triggered volume response of anionic hydrogel microspheres. Macromolecules 31: 5084-5093.

[9] Eichenbaum GM, Kiser PF, Dobrynin AV, Simon SA, Needham D (1999) Investigation of the swelling response and loading of ionic microgels with drugs and proteins: The dependence on cross-link density. Macromolecules 32: 4867-4878.

[10] Eichenbaum GM, Kiser PF, Shan D, Simon SA, Needham D (1999) Investigation of the swelling response and loading of ionic microgels with drugs and proteins: The dependence on functional group composition. Macromolecules 32: 8996-9006.

[11] Markland P, Zhang Y, Amidon GL, Yang VC (1999) A pH- and ionic strengthresponsive polypeptide hydrogel: synthesis, characterization, and preliminary protein release studies. J. Biomed. Mater. Res. 47: 595-602.

[12] Tanaka T, Nishio I, Sun ST, Nishio SU (1982) Collapse of gels in electric field. Science 218: 467-469.

[13] Suzuki B, Tanaka T (1990) Phase transition in polymer gels induced by visible light. Nature 346: 345-347. 
[14] Miyata T, Asami N, Uragami T (1999) A reversibly antigen-responsive hydrogel. Nature 399: 766-769.

[15] Kazakov S (2007) Phase transitions in smart polymer solutions and light scattering in biotechnology and bioprocessing. In: Galaev I, Mattiasson B, editors. SMART POLYMERS: Application in biotechnology and biomedicine, Taylor \& Francis: Chapter 1, pp $1-52$.

[16] Gehrke S (1993) Synthesis, equilibrium swelling, kinetics, permeability and applications of environmentally responsive gels. Adv. Polym. Sci. 110: 81-144.

[17] Peppas NA (2007) Drug delivery using smart polymers: Recent advances. In: Galaev I, Mattiasson B, editors. SMART POLYMERS: Application in biotechnology and biomedicine, Taylor \& Francis: Chapter 11, pp. 331-358.

[18] Kabra BG, Akhtar MK, Gehrke SH (1992) Volume change of poly(Nisopropylacrylamide) gels. Polymer Int. 28: 29-36.

[19] Harsh D, Gehrke SJ (1991) Controlling the swelling characteristics of temperaturesensitive cellulose ether hydrogels, Journal of Controlled Release 17: 175-186.

[20] McPhee W, Tam KC, Pelton R J (1993) Poly(N-isopropylacrylamide) latexes prepared with SDS. J. Colloid Interface Sci. 156: 24-30.

[21] Hirotsu S, Hirokawa Y, Tanaka T (1987) Volume-phase transition of ionized Nisopropylacrylamaide. J. Chem. Phys. 87: 1392-1395.

[22] Zhou Sh, Chu B (1998) Synthesis and volume phase transition of poly(methacrylic acid-co-N-isopropylacrylamide) microgel particles in water. J. Phys. Chem. 102: 1364-1371.

[23] Shibayama M, Mizutani S, Nomura S (1996) Thermal Properties of Copolymer Gels Containing N-Isopropylacrylamide. Macromolecules 29: 2019-2024.

[24] Hirose T, Amiya T, Hirokawa Y, Tanaka T (1987) Phase transition of submicron gel beads. Macromolecules 20: 1342-1344.

[25] Kokufuta E, Wang B, Yoshida R, Khokhlov A, Hirata M (1998) Volume phase transition of polyelectrolyte gels with different charge distributions. Macromolecules 3: 6878-6884.

[26] Kazakov S, Kaholek M, Gazaryan I, Krasnikov B, Miller K, Levon K (2006) Ion concentration of external solution as a characteristic of micro- and nanogel ionic reservoirs. J. Phys. Chem. 110: 15107-15116

[27] Ogawa Y, Ogawa K, Wang B, Kokufuta E (2001) A biochemo-mechanical system consisting of polyampholyte gels with coimmobilized glucose oxidase and urease. Langmuir 17: 2670-2674.

[28] Eremeev NL, Kukhtin AV, Kazanskaya NF (1998) Enzyme-dependent responses of stimuli-sensitive systems. Biosystems 45: 141-149.

[29] Horkay F, Tasaki I, Basser PJ (2001) Effect of monovalent-divalent cation exchange on the swelling of polyacrylate hydrogels in physiological sait solutions. Biomacromolecules 2: 195-199. 
[30] Horkay F, Tasaki I, Basser PJ (2000) Osmotic pressure of polyacrylate hydrogels in physiological salt solutions. Biomacromolecules 1: 84-90.

[31] Eichenbaum GM, Kiser PF, Shah D, Meuer WP, Needham D, Simon SA (2000) Alkali earth metal binding properties of ionic microgels. Macromolecules 33: 4087-4093.

[32] Amiya T, Tanaka T (1987) Phase transitions in cross-linked gels of natural polymers. Macromolecules 20: 1162-1164.

[33] Horkay F, Tasaki I, Basser PJ (2001) Ion-induced volume transition in synthetic and biopolymer gels. Polymer Preprint 42(1): 267-268.

[34] Iwasa K, Tasaki I (1980) Mechanical changes in squid giant axons associated with production of action potentials. Biochem. Biophys. Res. Commun. 95: 1328-1331.

[35] Tasaki I, Iwasa K (1982) Rapid pressure changes and surface displacements in the squid giant axon associated with production of action potentials. Jpn. J. Physiol. 32: 69-81.

[36] Tasaki I, Nakaye T, Byrne PM (1985) Rapid swelling of neurons during synaptic transmission in the bullfrog sympathetic ganglion. Brain Res. 331: 363-365.

[37] Tasaki I, Byrne PM (1988) Large mechanical changes in the bullfrog olfactory bulb evoked by afferent fiber stimulation. Brain Res. 475: 173-176.

[38] Tasaki I, Byrne PM (1992) Discontinuous volume transitions in ionic gels and their possible involvement in the nerve excitation process. Biopolymers 32: 1019-1023.

[39] Tasaki I, Byrne PM (1994) Discontinuous volume transitions induced by calciumsodium ion exchange in anionic gels and their neurobiological implications. Biopolymers 34: 209-215.

[40] Tasaki I (1999) Rapid structural changes in nerve fibers and cells associated with their excitation processes. Jpn. J. Physiol. 49: 125-138.

[41] Kokufuta E, Zhang YQ, Tanaka T, Mamada A (1993) Effects of surfactants on the phase transition of poly(N-isopropylacrylamide) gel. Macromolecules 26: 1053-1059.

[42] Khokhlov AR, Kramarenko EYu, Makhaeva EE, Starodubtsev SG (1992) Collapse of Polyelectrolyte Networks Induced by Their Interaction with Oppositely Charged Surfactants Macromolecules 25: 4779-4783.

[43] Gong JP, Osada Y (1995) Theoretical analysis of the cross-linking effect on the polyelectrolyte-surfactant interaction. J. Phys. Chem. 99: 10971-10975.

[44] Safranj A, Yoshida M, Omichi H, Katakai R (1994) Effect of surfactants on the volume phase transition of cross-linked poly(acryloyl-1-proline alkyl esters). Langmuir 10: 29552959.

[45] Yoshida M, Asano M, Omichi H, Kamimura W, Kumakura M, Katakai R (1997) Dependence of volume phase transition temperature of poly(acryloyl-L-proline methyl ester) gel on hydrophobic tail length of anionic surfactants. Macromolecules 30: 27952796.

[46] Okuzaki H, Osada Y (1994) Effects of hydrophobic interaction on the cooperative binding of a surfactant to a polymer network. Macromolecules 27: 502-506. 
[47] Murase Y, Onda T, Tsujii K, Tanaka T (1999) Discontinuous binding of surfactants to polymer gel resulting from a volume phase transition. Macromolecules 32: 85898594.

[48] Ogawa K, Ogawa Y, Kokufuta E (2002) Effect of charge inhomogeneity of polyelectrolyte gels on their swelling behavior. Colloids and Surfaces 209: 267-279.

[49] Okuzaki H, Osada Y (1994) Effects of hydrophobic interaction on the cooperative binding of a surfactant to a polymer network. Macromolecules 27: 502-506.

[50] Narita T, Gong JP, Osada Y (1998) Kinetic study of surfactant binding into polymer gel - Experimental and theoretical analysis. J. Phys. Chem. B. 102: 4566-4572.

[51] Akira Mamada A, Tanaka T, Kungwatchakun D, Irie M (1990) Photoinduced Phase Transition of Gels. Macromolecules 23: 1517-1519.

[52] Suzuki A, Tanaka T (1990) Phase transitions in polymer gels induced by visible light. Nature 346: 345-347.

[53] Juodkazis S, Mukai N, Wakaki R, Yamaguchi A, Matsuo Sh, Misawa H (2000) Reversible phase transitions in polymer gels induced by radiation forces. Nature 408: 178-181.

[54] Lendlein A, Jiang H, Junger O, Langer R (2005) Light-induced shape- memory polymers. Nature 434: 879-882.

[55] Kuhn W, Hargitay B, Katchalsky A, Eisenberg H (1950) Reversible Dilation and Contraction by Changing the State of Ionization of High-Polymer Acid Networks. Nature 165: 514-516.

[56] Steiberg IZ, Oplatka H, Katchalsky A (1966) Mechanochemical Engines. Nature 210: 568-571.

[57] Sussman MV, Katchalsky A (1970) Mechanochemical turbine: a new power cycle. Science 167: 45-47.

[58] Osada Y, Hasebe M (1985) Electrically activated mechanochemical devices using polyelectrolyte gels. Chem. Lett. 1285-1288.

[59] DeRossi DE, Chiarelli P, Buzzigoli G, Domenci C, Lazzeri L (1986) Contractile behaviour of electrically activated mecltanochemical polymer actuators. Trans. Am. Soc. Artif. Organs 32: 157-162.

[60] Tanaka T, Nishio I, Sun ST, Nishio SU (1982) Collapse of gels in an electric field. Science 218: 467- 469.

[61] Kishi R, Osada Y (1989) Reversible volume change of microparticles in an electric field. J. Chem. Soc., Faraday Trans. 1 85: 655-662

[62] Katchalsky A, Steinberg IZ, Kam A (1967) Mechanochemical engine. US Patent 3321908.

[63] Osada Y, Rossmurphy SB (1993) Intelligen gels. Scientific American 268: 82-87.

[64] Schreyer HB, Gebhart N, Kim KJ, Shahinpoor M (2000) Electrical activation of artificial muscles containing polyacrylonitride gel fibers. Biomacromolecules 1: 642 -647.

[65] Onoda M, Kato Y, Shonaka H, Tada K (2004) Artificial muscle using conducting polymers. Electrical Eng. Jpn. 149: 7-13. 
[66] Grodzinsky AJ, Grimshaw PE (1990) Electrically and chemically controlled hydrogels for drug delivery. Pulsed and Self-regulated Drug Delivery pp. 47-64.

[67] Moorthy J (2007) Hydrogels in microfluidics. In: Galaev I, Mattiasson B, editors. SMART POLYMERS: Application in biotechnology and biomedicine, Taylor \& Francis: Chapter 14, pp. 437-457.

[68] Lee KY, Mooney DJ (2001) Hydrogels for tissue engineering. Chemical Reviews 101: 1869-1879.

[69] Schwall CT, Banerjee IA (2009) Micro- and nanoscale hydrogel systems for drug delivery and tissue engineering. Materials 2: 577-612.

[70] Schmaljohann D, Nitschke M, Schulze R, Eing A, Werner C, Eichhorn K-J (2005) In situ study of the thermoresponsive behavior of micropatterned hydrogel films by imaging ellipsometry. Langmuir 21: 2317-2322.

[71] Green PG (1996) Iontophoretic delivery of peptide drugs. J. Control. Release 41: 3348.

[72] Thompson LF, Wilson CG, Bowden MJ, Eds. (1994) Introduction to Microlithography, American Chemical Society.

[73] Levenson MD (1995) Extending optical lithography to the gigabit era. Solid State Technol. February: 57-66

[74] Pease RFW (1992) Nanolithography and its prospects as a manufacturing technology. J. Vac. Sci. Technol. B 10: 278-285

[75] Cerrina F, Marrian C (1996) A path to nanolithography. Mater. Res. Soc. Bull. XXI: 56-62

[76] Ecoffet C, Espanet A, Lougnot DJ (1998) Photopolymerization by evanescent waves: A new method to obtain nanoparts. Adv. Mater. 10: 411-414.

[77] Yariv A (1989) Quantum Electronics. $3^{\text {rd }}$ ed.; John Wiley \&Son.

[78] Mehrvar M, Bis C, Scharer JM, Moo-Young M, Luong JH (2000) Fiber-optics biosensors - Trends and advances. Analytical Sciences 16: 677-691.

[79] Wolfbeis OS (2004) Fiber-optic chemical sensors and biosensors. Anal. Chem. 76: 32693284.

[80] Yang J, Lee C-J, Wei C-H (2002) Fiber-optic chemical sensors: a general review. J. Chin. Chem. Soc. 49: 677-692.

[81] Steemers FJ, Walt DR (1999) Multi-analyte sensing: From site-selective deposition to randomly-ordered addressed optical sensors. Microchimica Acta 131: 99-105.

[82] Michie WC, Culshaw B, McKenzie I, Konstantakis M, Graham NB, Moran C, Bergquist E, Carlstrom B (1995) Distributed sensor for water and $\mathrm{pH}$ measurements using fiber optics and swellable polymeric systems. Optics Letters 20: 103-105.

[83] Rassell AP, Fletcher KS (1985) Optical sensor for the determination of moisture. Anal. Chim. Acta 170: 209-216.

[84] Hypszer R, Wierzba HJ (1997) Fiber optic technique for relative humidity sensors. SPIE Proceedings 3054: 145-150. 
[85] Wyatt WA, Bright FV, Hieftje GM (1987) Characterization and comparison of three fiber-optic sensors for iodide determination based on dynamic fluorescence quenching of Rhodamine 6G. Anal. Chem. 59: 2272-2276.

[86] Shortreed M, Bakker E, Kopelman R (996) Miniature sodium-selective ion-exchange optode with fluorescent $\mathrm{pH}$ chromoionophores and tunable dynamic range. Anal. Chem. 68: 2656-2662.

[87] Mohr GJ, Wolfbeis (1996) Optical nitrite sensor based on a potential-sensitive dye and a nitrite-selective carrier. Analyst 121: 1489-1494.

[88] Hartman P, Trettnak W (1996) Lifetime imaging of luminescent oxygen sensors based on all-solid-state technology. Anal. Chem. 68: 4512-4514.

[89] Ferguson JA, Healey BG, Bronk KS, Bonrnard SM, Walt DR (1997) Simultaneous monitoring of $\mathrm{pH}, \mathrm{CO}_{2}$ and $\mathrm{O}_{2}$ using an optical imaging fiber. Anal. Chim. Acta 340: 123131.

[90] Li L, Walt DR (1995) Dual-analyte fiber-optic sensor for the simultaneous and continuous measurement of glucose and oxygen. Anal. Chem. 67: 3746-3752.

[91] Shakhsher ZM, Seitz WR (1990) Optical detection of cationic surfactants based on ion pairing with an environment-sensitive fluorophore. Anal. Chem. 62: 1758-1762.

[92] Barnard SM, Walt DR (1991) A fibre-optic chemical sensor with discrete sensing sites. Nature 353: 338-340.

[93] Healey BG, Walt DR (1995) Improved fiber-optic chemical sensor for penicillin. Anal. Chem. 67: 4471-4476.

[94] Ge Z, Brown CW, Sun L, Yang SC (1993) Fiber-optic pH sensor based on evanescent wave absorption spectroscopy. Anal. Chem. 65: 2335-2338.

[95] Kazakov S (2010) Evanescent wave spectroscopy for studying swelling/de-swelling kinetics of soft matter. Soft Matter 6: 1191-1196.

[96] Wang D, Elisseeff JH (2004) Photopolymerization. In Encyclopedia of Biomaterials and Biomedical Engineering (EBBE), Wnek, GE, Bowlin GL, Eds: Marcel Dekker, Inc.: pp 1212-1225.

[97] Belfield KD, Crivello JV (1996) Photoinitiated polymerization. American Chemical Society.

[98] Decker C, Jenkins AD (1985) Kinetic approach of $\mathrm{O}_{2}$ inhibition in ultraviolet- and laserinduced polymerization. Macromolecules 18: 1241-1244.

[99] Lee JH, Prud'homme RK, Aksay IA (2001) Cure depth in photopolymerization: Experiments and theory. J. Mater. Res. 16: 3536-3544.

[100] Geuskens G, Soukrati A (2000) Investigation of polyacrylamide hydrogels using 1-anilinonaphthalene-8-sulfonate as fluorescent probe. Eur. Polymer J. 36: 15371546.

[101] Thomas TL, Mishra AK (2002) ANS fluorescence as a tool to monitor cross-linking polymerization of acrylamide. Eur. Polymer J. 38: 1805-1810.

[102] Kazakov S, Kaholek M, Kudasheva D, Teraoka I, Cowman KM, Levon K (2003) Poly(N-isopropylacrylamide-co-1-vinylimidazole) hydrogel nanoparticles prepared 
and hydrophobically modified in liposome reactors: Atomic force microscopy and dynamic light scattering study. Langmuir 19: 8086-8093.

[103] Kazakov S, Kaholek M, Teraoka I, Levon K (2002) UV-induced gelation on nanometer scale using liposome reactor. Macromolecules 35: 1911-1920.

[104] Kazakov S, Kaholek M, Krasnikov B, Levon K (2003) Approach to study pHresponsive micro- and nanogels. Polym. Preprint 44: 186-187.

[105] Kazakov S, Kaholek M, Levon K (2002) Nanometer scale ionic reservoir based on ionresponsive hydrogels. SPIE Proceedings 4695: 42-51.

[106] Kazakov S, Kaholek M, Levon K (2002) Hydrogel nanoparticles compatible with phospholipid bilayer. Polym. Preprint 43: 381-382.

[107] Munkholm C, Parkinson D-R, Walt DR (1990) Intramolecular fluorescence selfquenching of fluoresceinamine. J. Am. Chem. Soc. 112: 2608-2612 and references therein.

[108] Berney C, Danuser G (2003) FRET or no FRET: A quantitative comparison. Biophys. J. 84: 3992-4010 and references within.

[109] Molecular Probes Publications online: www.invitrogen.com/handbook/

[110] Schild HG (1992) Poly(N-isopropilacrylamide): experiment, theory and application. Prog. Polym. Sci. 17: 163-249.

[111] Ulijn RV, Bibi N, Jayawarna V, Thornton PD, Todd SJ, Mart RJ, Smith AM, Gough JE (2007) Materials Today 10: 40-48.

[112] Aldrich Polymer Products Application and Reference Information, Aldrich Chemical Co. online Publication, 1-52, www.sigmaaldrich.com

[113] Zhang S, Li B, Tang L, Wang X, Lui D, Zhou Q (2001) Studies on the near infrared laser induced photopolymerization employing a cyanine dye-borate complex as a photoinitiator. Polymer 42: 7575-7582.

[114] Warken F, Vetsch E, Meschede D, Sokolowski M, Rauschenbeutel A (2007) Ultrasensitive surface absorption spectroscopy using sub-wavelength diameter optical fiber. Optics Express 15: 11952-11958.

[115] Wu H-J, Shah S, Beckham R, Meissner KE, and Michael A. Bevan MA (2008) Resonant effects in evanescent wave scattering of polydisperse colloids. Langmuir 24: 1397013795.

[116] Trutna R, Liu M, Chamberlin D, Hadley J (2008) Nanoparticle measurement by spectroscopic Mie scattering. NSTI-Nanotech 1: 834-837.

[117] De SK, Aluru NR, Johnson B, Crone WC, Beebe DJ, Moore J (2202) Equilibrium swelling and kinetics of pH-responsive hydrogels: Models, experiments, and simulations. Journal of Microelectromechanical Systems 11: 544-555.

[118] Plunkett KN, Kraft ML, Yu Q, Moore JS (2003) Swelling kinetics of disulfide crosslinked microgels. Macromolecules 36: 3960-3966.

[119] Kazakov S, Kaholek M, Ji T, Turnbough C, Levon K (2004) Interaction of modified liposomes with Bacillus spores. Chem. Commun. 4: 430-431. 
[120] Simpson ML, Sayler GS, Fleming JT, Applegate B (2001) Whole-cell biocomputing. Trends Biotech. 19: 317-323.

[121] Wagner ML, Tamm LK (2000) Tethered polymer-supported planar lipid bilayers for reconstitution of integral membrane proteins: silane-polyethyleneglycol-lipid as a cushion and covalent linker. Biophys. J. 79: 1400-1414.

[122] Zhao J, Tamm LK (2003) FTIR and fluorescence studies of interactions of synaptic fusion proteins in polymer-supported bilayers. Langmuir 19: 1838-1846.

[123] Kiessling V, Tamm LK (2003) Measuring distances in supported bilayers by fluorescence interference-contrast microscopy: polymer supports and SNARE proteins. Biophys. J. 84: 408-418.

[124] Sackmann E, Tanaka M (2000) Supported membranes on soft polymer cushions: fabrication, characterization and applications. Trends Biotech. 18: 58-64.

[125] Heyse S, Ernst OP, Dienes Z, Hofmann KP, Vogel H (1998) Incorporation of rhodopsin in laterally structured supported membranes: observation of transducin activation with spatially and time-resolved surface plasmon resonance. Biochemistry 37: 507-522

[126] Parsek MR, Fuqua C (2004) Biofilms 2003: Emerging themes and challenges in studies of surface-associated microbial life. J. Bacteriology 186: 4427-4440.

[127] Foster SJ, Johnstone K (1989) The trigger mechanism of bacterial spore germination. In book: Regulation of prokaryotic development. I. Smith, R.A. Slepecky, P. Setlow (Eds.), Ch. 4, ASM, Washington, DC.

[128] Swerdlow BM, Setlow B, Setlow P (1981) Level of $\mathrm{H}^{+}$and other monovalent cations in dormant and germinating spores of Bacillus megaterium. J. Bacteriology 148: 26-29.

[129] Makino S, Moriyama R (2002) Hydrolysis of cortex peptidoglycan during bacterial spore germination. Med. Sci. Monit. 8: RA119-127.

[130] Hersel U, Dahmen C, Kessler H (2003) RGD-modified polymers: biomaterials for stimulated cell adhesion and beyond. Biomaterials 24: 4385-4415.

[131] Zourob M, Gough JE, Ulijn RV (2006) A micro-patterned hydrogel platform for chemical synthesis and biological analysis. Adv. Mater. 18: 655-659.

[132] Silva GA, Czeisler C, Niece KL, Beniash E, Harrington DA, Kessler JA, Stupp SI (2004) Selective differentiation of neural progenitor cells by high-epitope density nanofibers. Science 303: 1352-1355.

[133] Rajangam K, Behanna HA, Hui MJ, Han X, Hulvat JF, Lomasney JW, Stupp SI (2006) Heparin Binding Nanostructures to Promote Growth of Blood Vessels. Nano Lett. 6: 2086-2090.

[134] Lutolf MP, Hubbell JA (2005) Synthetic biomaterials as instructive extracellular microenvironments for morphogenesis in tissue engineering. Nat. Biotechnol. 23: 4755

[135] Stevens MM, George JH (2005) Exploring and engineering the cell surface interface. Science 310: 1135-1138. 
[136] Langer R, Tirrell DA (2004) Designing materials for biology and medicine. Nature 428: 487-492.

[137] Duncan R (2003) The dawning era of polymer therapeutics. Nat. Rev. Drug Discov. 2: 347-360.

[138] Lee KY, Mooney DJ (2001) Hydrogels for tissue engineering. Chem. Rev. 101: 18691879. 